\title{
Akkermansia Muciniphila and Environmental Enrichment Reverse Cognitive Impairment Associated With High-fat High-cholesterol Consumption Through the Gut-brain Axis
}

\section{Sara G. Higarza}

University of Oviedo

\section{Silvia Arboleya}

Instituto de Productos Lacteos de Asturias

Jorge L. Arias

University of Oviedo

Miguel Gueimonde ( $\nabla$ mgueimonde@ipla.csic.es )

Instituto de Productos Lacteos de Asturias

Natalia Arias ( $\square$ natalia.arias@kcl.ac.uk)

King's College London https://orcid.org/0000-0001-5602-5854

\section{Research}

Keywords: NASH, cognition, novel object recognition, working memory, gut microbiota, microbiota-gutbrain axis, environmental enrichment, probiotics

Posted Date: June 9th, 2020

DOI: https://doi.org/10.21203/rs.3.rs-33859/v1

License: (c) (i) This work is licensed under a Creative Commons Attribution 4.0 International License.

Read Full License 


\section{Abstract}

Background: Non-alcoholic steatohepatitis (NASH) is one of the most prevalent diseases worldwide. A high-fat, high-cholesterol (HFHC) diet leads to an early NASH model. It has been suggested that gut microbiota mediates the effects of diet through the microbiota-gut-brain axis, modifying the host's brain metabolism and disrupting cognition.

Results: Here, we target NASH-induced cognitive damage by testing the impact of environmental enrichment (EE) and the administration of either Lacticaseibacillus rhamnosus GG (LGG) or Akkermansia muciniphila CIP107961 (AKK). EE and AKK, but not LGG, reverse the HFHC-induced cognitive dysfunction, including impaired spatial working memory and novel object recognition; however, whereas AKK restores brain metabolism, EE produces an overall decrease. Moreover, AKK and LGG did not induce major rearrangements in the intestinal microbiota, with only slight changes in bacterial composition and diversity, whereas EE entailed an increase in Firmicutes and Verrucomicrobia members.

Conclusions: Our findings illustrate the interplay between gut microbiota, the host's brain energy metabolism, and cognition, and they suggest intervention strategies, such as the administration of AKK, for the management of the cognitive dysfunction related to NASH.

\section{Background}

Non-alcoholic fatty liver disease (NAFLD) is characterised by hepatic fat accumulation, and it is closely associated with central obesity, diabetes, and other features of metabolic syndrome [1]. NAFLD encompasses a broad disease spectrum ranging from simple steatosis to non-alcoholic steatohepatitis (NASH), which may, in turn, develop into cirrhosis, end-stage liver disease, or hepatocarcinoma [2]. Although NASH has become a serious global health threat, therapeutic strategies that could prevent NAFLD-NASH progression have been overlooked.

We previously published [3] a study showing that consumption of a high-fat, high-cholesterol diet leads to an early NASH model that replicates the typical features of the human disease, including hepato- and splenomegaly, early NASH histopathology, hypercholesterolemia, increased serum liver enzymes, and increased pro-inflammatory cytokines $[3,4]$. Studies have reported that a high-fat, high-cholesterol diet and a high-fat diet produce changes in the gut microbial composition, as well as reductions in microbial diversity and changes in specific bacterial taxa $[3,5,6]$. In this line, a recent study by Hoyles et al. [7] showed that faecal transplantation from human donors with hepatic steatosis triggered rapid development of hepatic steatosis in mice, which highlights the contributing role of microbiota in NASH development.

Gut microbiota has also been found to play an important role in the central nervous system (CNS) through the microbiota-gut-brain axis, and CNS dysfunction has been linked to several psychiatric and non-psychiatric disorders [8]. The involvement of gut microbiota dysbiosis and altered short-chain fatty acid (SCFAs) production has been proposed in neurodegenerative disorders such as Parkinson's disease 
[9]. Recent studies have also reported altered microbiota in autism spectrum disorder, anxiety, or myasthenia gravis, among other neurological conditions [8, 10-12]. Moreover, in a previous study we observed gut microbiota dysbiosis and microbial metabolite alterations linked to cognitive impairment in a NASH rat model [3]. These findings highlight the functional connection between the liver-gut microbiota and cognition. Thus, gut microbiota could be a target for improving cognitive deficiencies associated with $\mathrm{NASH}$, with probiotics as a promising tool for this purpose. However, the impact of probiotic administration on cognitive function is not well understood.

Furthermore, an inappropriate diet and physical inactivity often co-exist in these patients. Exercise is able to modulate the gut microbiota. Clarke et al. [13] described that athletes showed a higher diversity of gut microorganisms, an observation also supported by other studies $[14,15]$. In the brain, studies in elderly people have shown that exercise increases hippocampal volume and enhances hippocampus-dependent learning and memory [16]. The effect of physical exercise in rodents is modelled using environmental enrichment (EE), which induces a situation of increased motor stimulation and sensorial and cognitive enhancement [17]. EE has been shown to be associated with synaptic function and cellular plasticity changes, resulting in cognitive enhancement [17-19]. Therefore, EE could mitigate the negative impact of a high-fat, high-cholesterol diet on the brain and cognition.

In the present study, we target high-fat, high-cholesterol (HFHC) induced cognitive disturbances through the microbiota-gut-brain axis using two different approaches. First, we assess the effects of EE as a cognitive enhancer, and, second, we evaluate the impact of two specifically selected strains of probiotics, Lacticaseibacillus rhamnosus GG (LGG) and Akkermansia muciniphila CIP107961 (AKK), on NASH pathology. In both cases, we compare their effects on microbiota changes, brain metabolism, and, ultimately, cognitive improvement.

\section{Methods}

Experimental groups. Fifty-six male Sprague-Dawley rats (220 $\mathrm{g}$ at the start) (Envigo, United Kingdom) were divided into seven groups ( $n=8$ per experimental group): $N C$ (normal chow), $N C+E E$ (normal chow + environmental enrichment), HFHC (high-fat, high cholesterol diet), HFHC + EE, HFHC + PBS (phosphatebuffered saline), HFHC + LGG (Lacticaseibacillus rhamnosus GG, ATCC53103) and HFHC + AKK (Akkermansia muciniphila CIP107961). Normal chow contained $13 \mathrm{kcal} \%$ from fat and no cholesterol (Envigo, United Kingdom - 2914), and NASH was induced through a HFHC diet (Research Diets, USA D09052204) containing $65 \mathrm{kcal} \%$ from fat and $2 \mathrm{kcal} \%$ cholesterol. All the groups were weighed weekly and studied after 14 weeks of the administration of the diet (Fig. 1a). Each subject was subjected to a different intervention and performed the behavioural tests only once. The animals had ad libitum tap water and were maintained at constant room temperature $\left(22 \pm 2{ }^{\circ} \mathrm{C}\right)$, with a relative humidity of $65 \pm 5 \%$ and an artificial light-dark cycle of $12 \mathrm{~h}(08: 00-20: 00 / 20: 00-08: 00 \mathrm{~h})$.

Environmental enrichment. The $\mathrm{NC}+\mathrm{EE}$ and $\mathrm{HFHC}+\mathrm{EE}$ groups were continuously submitted to $\mathrm{EE}$ from week 8 to week 12 (Fig. 1a). During this time, each experimental group was separately housed in groups 
of 8 subjects in large cages measuring $76.5 \mathrm{~cm} \times 48 \mathrm{~cm} \times 81 \mathrm{~cm}$, maintaining the same previous contingencies in terms of food, water administration, and room conditions. The cages contained different stimulating objects, including platforms, tubes, little houses, running wheels, balls, and toys made of different materials, textures, shapes, sizes, and colours [20]. The stimulating objects were the same in both groups, and they were changed weekly to ensure novelty.

Probiotics culture and administration. Two different bacterial strains were used, L. rhamnosus GG (ATCC 53103) (LGG) and A. muciniphila CIP107961 (AKK). They were selected based on previous studies carried out by the group. First, in our previous characterization of the NASH animal model [3], we observed that the gut microbiota in NASH rats suffered an important loss of Lactobacillus compared to the NC group. Because of this, we decided to test a Lactobacillus strain as a potential probiotic, and we selected the widely used probiotic strain L. rhamnosus GG [21], which has previously been demonstrated to positively modulate liver fatty acid composition in mice receiving a high-fat diet [22]. Second, in a previous pilot study, we tested the effect of EE on the gut microbiota and cognitive aberrances of a pilot NASH rat model (data not shown). We observed that EE increased the levels of Akkermansia in the HFHC + EE group and ameliorated the NASH symptoms, compared to the HFHC control group. Therefore, we also decided to include the potentially novel probiotic Akkermansia muciniphila, which has been found in different animal models to ameliorate metabolic syndrome features such as obesity, diabetes, and cardiovascular diseases [23], enhance the lifespan in a progeria mice model [24], or improve amyotrophic lateral sclerosis symptoms [25]. Moreover, in a recent human intervention study, A. muciniphila was found to improve liver function and metabolic markers [26].

L. rhamnosus GG was grown in a de Man, Rogosa and Sharpe (MRS) medium (Difco, Becton Dickinson), and A. muciniphila CIP107961 was grown in a GAM medium (Nissui Pharmaceutical Co.). Both were incubated at $37^{\circ} \mathrm{C}$ in an anaerobic chamber (Mac 500; Don Whitley Scientific) under a $10 \%$ (v/v) H2, $10 \%$ $(\mathrm{v} / \mathrm{v}) \mathrm{CO} 2$ and $80 \%(\mathrm{v} / \mathrm{v}) \mathrm{N} 2$ atmosphere. Twenty-four hour culture was used to inoculate $(1 \% \mathrm{v} / \mathrm{v})$ fresh MRS broth or a pre-reduced GAM broth medium, which were incubated overnight (L. rhamnosus $\mathrm{GG}$ ) and for $24 \mathrm{~h}$ (A. muciniphila CIP107961). Then, the cultures were washed and concentrated with pre-reduced PBS, which included $20 \%(\mathrm{v} / \mathrm{v})$ glycerol to a concentration of about $1 \times 10^{10} \mathrm{cfu} / \mathrm{ml}$ and was stored at $-80^{\circ} \mathrm{C}$ until administration. The HFHC + LGG and HFHC + AKK animals were administered their respective bacteria at a final concentration of $1 \times 10^{9} \mathrm{cfu}$. Before gavage, glycerol stocks were thawed under anaerobic conditions, centrifuged to remove glycerol, and resuspended in $1 \mathrm{ml}$ of pre-reduced PBS. HFHC + LGG and HFHC + AKK rats were administered $100 \mu \mathrm{l}$ of L. rhamnosus GG and A. muciniphila CIP107961, respectively, and a control group received $100 \mu$ l of PBS (HFHC + PBS) daily from weeks 8 to 12 of the HFHC diet (Fig. 3a).

Viability of the glycerol stocks was tested by serial dilutions in PBS and plating counts in MRS (48 h) and GAM-agar (5 days), respectively, and incubation under anaerobic conditions. Both strains were validated for purity by whole-gene $16 \mathrm{~S}$ Sanger sequencing. 
Faecal sample collection and processing. Fresh faecal pellets from each animal were collected and kept at $-80^{\circ} \mathrm{C}$ until DNA extraction. Before that, samples were weighed, diluted in PBS $(1: 5 \mathrm{w} / \mathrm{v})$, and homogenized (3 min, full speed) in a stomacher (LabBlender 400). From $1 \mathrm{~mL}$ of the homogenate, faecal supernatant and cellular pellets were separated by centrifugation (10.000 rpm, $15 \mathrm{~min})$ and stored at $-20^{\circ} \mathrm{C}$. The supernatant was used for SCFA and BCFA analysis, and the pellets were used for DNA extraction with the QIAamp DNA stool kit (Qiagen, GmbH, Germany), as described elsewhere [27].

Analysis of faecal microbial groups by $16 \mathrm{~S}$ rRNA gene profiling and quantitative PCR. Extracted Isolated DNA was used as a template for amplification of the $\mathrm{V} 3$ region from partial 16S rRNA gene sequences by PCR, using the primers and conditions described by Milani and coworkers [28]. The amplicons obtained were then sequenced by using the MiSeq (Illumina) platform) at GenProbio srl (Italy). The individual reads obtained were filtered, trimmed, and processed [29]. 16S rRNA Operational Taxonomic Units were defined at $\geq 97 \%$ sequence homology using the uclust tool developed by Edgar[30]. All reads were classified in the lowest possible taxonomic rank using QIIME 2 and a reference dataset from the SILVA database [31]. Akkermansia and Lactobacillus genus were also determined by quantitative PCR using the previously described primers and conditions [32].

Determination of short-chain and branched-chain fatty acids in faeces. SCFA and BCFA levels were determined in the faecal supernatants by means of gas chromatography, as described by Moris et al. [11]. Briefly, $250 \mu \mathrm{l}$ of cell free-supernatants were mixed with $100 \mu \mathrm{l}$ methanol, $50 \mu \mathrm{l}$ internal standard solution (2-ethylbutyric $1.05 \mathrm{mg} / \mathrm{ml}$ ), and $50 \mu \mathrm{l}$ of $20 \% \mathrm{v} / \mathrm{v}$ formic acid. The mix was then centrifuged, and the supernatant was injected into a system composed of a 6890NGC injection module (Agilent Technologies Inc., USA) connected to a flame injection detector (FID) and a mass spectrometry detector (MS, 5973N) (Agilent) for quantification of both SCFA and BCFA.

Locomotor activity assessment. The study of locomotor activity was carried out by using Rotarod 7750 for rats, which consists of a motor-driven rotating rod (Ugo Basile Biological Research Apparatus) [33]. After habituation for $1 \mathrm{~min}$ at a constant speed of $2 \mathrm{rpm}$, the rats were evaluated for $5 \mathrm{~min}$, during which the speed increased constantly until $20 \mathrm{rpm}$. Rotation speed was recorded and used as a measure of locomotor function.

Novel object recognition. The object recognition test was carried out in an open field $(66 \times 46 \times 45 \mathrm{~cm})$ made of grey fibreglass, with an open roof. The open field was situated in a room with two diffuse white lights on its sides, providing an illumination density of 50 lux at its centre. The animal's behaviour was recorded by a video camera (Sony V88E) connected to a computer equipped with a computerised videotracking system (EthoVision Pro, Noldus Information Technologies, The Netherlands). After each trial, the apparatus was thoroughly cleaned with a $75 \%$ ethanol solution. The objects used were constructed from a combination of plastic pieces of different colours and shapes. The animals were habituated for two days before the test. On the first day, the animals were situated first in groups and then individually in the open field for two trials lasting 6 minutes each. On the second day, each animal was placed in the field, with two equal objects in the centre, for 3 trials lasting 6 minutes each, separated by 20 minutes. 
The test was composed of two phases. In the first one, in the open field, each subject was exposed for 4 minutes to two copies of a new object $(2 \mathrm{x}$ object $\mathrm{A})$, located in opposite corners at $10 \mathrm{~cm}$ from the walls. After a 50-minute delay, each animal carried out the second trial, which was similar to the previous one, except that a copy of the previously-encountered object was changed to a novel one (object A + object B).

Exploration of an object was defined as directing the nose towards the object at less than $2 \mathrm{~cm}$ and exploring it (i.e. sniffing and or interacting with the object). For each animal, time spent exploring the objects in both trials was measured. The proportion of exploration in the first (e1) and second (e2) phases of the test was considered as the ratio between the exploration of all objects in each phase and the total time available for exploration. The discrimination of the objects was assessed through the discrimination index (d1), the difference in time spent exploring the two objects in the second phase (novel minus familiar), and the discrimination ratio (d2), the difference in time spent exploring the two objects in the second phase divided by the total time exploring both objects [34].

Spatial working memory. Spatial working memory was measured in the Morris water maze (MWM), which is a cylindrical fibreglass tank, virtually divided into four quadrants, measuring $150 \mathrm{~cm}$ in diameter with a $40-\mathrm{cm}$ high wall [35]. The water level was $30 \mathrm{~cm}$, and its temperature was $22 \pm 2{ }^{\circ} \mathrm{C}$. The pool contained a cylindrical platform that was $10 \mathrm{~cm}$ in diameter and $28 \mathrm{~cm}$ high, of which $2 \mathrm{~cm}$ was below the surface and used by the animals to escape. The MWM was in the centre of a $16 \mathrm{~m}^{2}$ lit room (two halogen lamps of 4000 lx), surrounded by panels on which several extra-maze clues were placed. The animal's behaviour was recorded by a video camera (Sony V88E) connected to a computer equipped with a computerised video-tracking system (EthoVision Pro, Noldus Information Technologies, The Netherlands). One day before the test, the animals were habituated to the task in 3 trials with the platform, using different starting positions in a small square water tank $(47 \times 75 \times 38 \mathrm{~cm})$.

The spatial working memory test is a paired sample task carried out for five days. Each session consisted of two trials (sample and retention). Within the same session, the platform, which was invisible to the animals because it was hidden underwater, was situated in one of the four quadrants and the animal started facing the wall of another quadrant. The trial ended once the animal had found the platform, or when $60 \mathrm{~s}$ had elapsed. If the animal had not reached the hidden platform after this time, it was placed on the platform for $15 \mathrm{~s}$. During the intertrial interval, the animals were placed in a bucket for $5 \mathrm{~s}$. The quadrant containing the platform and the animal's starting point changed between sessions. Latencies to reach the escape platform were recorded and used as a measure of task acquisition. The learning criterion was when the animals spent significantly less time in the retention trial than in the sample trial in each session [36].

Brain metabolic activity. Ninety minutes after the last session of the spatial working memory task, the animals were decapitated, their brains were frozen and subsequently sliced in $30 \mu \mathrm{m}$-thick brain coronal sections. The protocol followed was previously described by our group [37], and staining variability across different baths was controlled through sets of brain tissue homogenate standards of known CCO activity from rat brains cut at different thicknesses $(10,30,50$, and $70 \mu \mathrm{m})$ [38]. 
The CCO histochemical staining intensity was quantified by densitometric analysis, using a computerassisted image analysis workstation (MCID, Interfocus Imaging Ltd). The mean optical density (OD) of each region was measured in twelve readings that were averaged to obtain one mean per region for each animal. These OD values were then converted to CCO activity units ( $\mu \mathrm{mol}$ of cytochrome $\mathrm{C}$ oxidised $/ \mathrm{min} / \mathrm{g}$ tissue wet weight), determined by the enzymatic activity of the standards measured spectrophotometrically.

Neuronal metabolic activity was measured in selected brain regions anatomically defined according to Paxinos and Watson's atlas [39]. The regions of interest and their distance from bregma were: the prefrontal cortex $(+3.24 \mathrm{~mm}$ ) (prelimbic (PrL), infralimbic (IL) and cingulate (Cg) cortices), the dorsal striatum (dST) $(+1.56 \mathrm{~mm})$ and ventral striatum $(+1.56 \mathrm{~mm})$ (accumbens core (AcbC) and shell (AcbSh)), the thalamus $(-1.20 \mathrm{~mm}$ ) (anteromedial nucleus (AMT), anterodorsal nucleus (ADT), and anteroventral nucleus (AVT)), the amygdala $(-2.28 \mathrm{~mm})$ (central (CeA), basolateral (BLA) and lateral (LaA)), the dorsal hippocampus (-3.00 mm) (dentate gyrus (DG), CA1 and CA3 areas), and the perirhinal (PRh) and entorhinal (Ent) cortices $(-4.20 \mathrm{~mm})$.

Statistical analysis. Data derived from microbiota were analysed using the IBM SPSS Statistics Version 24.0 (IBM Corp., USA) software. Comparisons of the groups following normal distributions (alphadiversity) were performed using one-way ANOVAs, followed by Tukey's post-hoc analyses. For nonparametric distributions (qPCR, SCFAs and BCFAs data), the Kruskal-Wallis test was performed, followed by Dunn's post-hoc test. Differences in bacteria abundances were calculated using the linear discriminant analysis effect size (LEfSe) method [40].

Body weight, behavioural data, and CCO values were analysed with the SigmaStat 3.2 program (Systat, USA). Body weight was analysed through a two-way ANOVA (Group x Week), followed by a post-hoc Tukey test when appropriate. Maximum speed spent on the Rotarod was compared between groups by using a Kruskal-Wallis test. On the novel object recognition test, two-tailed paired $t$-tests were used to analyse the exploration between the two trials (e1 and e2), and Mann-Whitney Utests for independent samples were performed on $\mathrm{d} 1$ and $\mathrm{d} 2$ to find out whether the mean scores differed from zero (with chance performance indicating equal exploration of the two objects). Additionally, d2 values were compared between the groups using a one-way ANOVA. On the spatial working memory test, sample and retention trial latencies were compared within each group with a two-tailed paired $t$-test, and between groups with a one-way ANOVA. Brain metabolic activity was evaluated between the groups through oneway ANOVA. When normality or equal group variances failed, Kruskal-Wallis one-way analysis of variance on ranks was performed. Post-hoc multiple comparison analyses were carried out, when allowed, using the Tukey method. The results were considered statistically significant if $p<0.05$.

\section{Results}

Environmental enrichment restores cognitive deficits caused by the HFHC diet. No statistically significant differences were found in the body weight between groups throughout the 14 weeks of administration of 
the diet (Fig. 1a) $(p=0.306)$. As expected, significant differences between weeks were revealed $(p<$ 0.001), with normal weight gain according to age in both experimental groups (Fig. 1b). The normal increase in weight was accompanied by the absence of statistically significant locomotor deficits in all groups ( $p=0.151$; Fig. $1 \mathrm{c})$.

When the experimental groups performed the novel object recognition task, there were no statistically significant differences between $\mathrm{e} 1$ and $\mathrm{e} 2$ in any of the groups (NC: $p=0.153$; NC + EE: $p=0.157$; HFHC: $p$ $=0.895 ; \mathrm{HFHC}+\mathrm{EE}: p=0.106)$, which means that these animals spent a similar amount of time exploring in the first and second phases of the test. However, when we compared the discrimination ratios, we found that the NC group was able to discriminate between a previously-encountered object and a novel object because $\mathrm{d} 1$ and $\mathrm{d} 2$ were statistically higher than zero (d1: $p<0.001 ; \mathrm{d} 2: p<0.001$ ), whereas the HFHC group was not able to distinguish the new object ( $\mathrm{d} 1: p=0.442 ; \mathrm{d} 2: p=0.442)$. After the implementation of EE, the $\mathrm{NC}+\mathrm{EE}$ group maintained its recognition ability (d1: $p<0.001 ; \mathrm{d} 2: p<0.001)$, and we found that the HFHC + EE group was now able to recognise the novel object ( $\mathrm{d} 1: p=0.010 ; \mathrm{d} 2: p=$ 0.010). When we compared the discrimination ratio between the experimental groups, we found that the $\mathrm{NC}, \mathrm{NC}+\mathrm{EE}$, and $\mathrm{HFHC}+\mathrm{EE}$ groups showed statistically significant higher $\mathrm{d} 2$ than the HFHC group $(p=$ 0.003). The HFHC group initially displayed an object recognition impairment that was reversed by the introduction of EE (Fig. 1d).

To explore whether or not the EE improvement only occurred in a particular network, the experimental groups were tested on a spatial working memory task. Whereas the NC group was able to remember the position of the platform, showing a statistically significant lower latency in the retention trial compared to the sample trial $(p=0.003)$, the HFHC group did not show statistically significant differences between the sample and retention trials $(p=0.333)$. After $\mathrm{EE}$, the $\mathrm{NC}+\mathrm{EE}$ maintained their capacity to remember the position of the platform $(p=0.002)$, and HFHC + EE were now able to remember it as well $(p=0.002)$. Our results showed that the HFHC impaired spatial working memory was ameliorated by the EE. When comparing the mean latencies in each trial between groups, we observed that, although the sample latencies were statistically similar, $(p=0.941)$, the retention latencies differed $(p=0.008)$ because they were significantly higher in HFHC than in NC, NC + EE, and HFHC + EE (Fig. 1e).

Each group was fed their respective diet for 14 weeks. From week 8 to 12, NC+EE and HFHC+EE were subjected to $\mathrm{EE}$, whereas $\mathrm{HFHC}+\mathrm{LGG}$ and $\mathrm{HFHC}+\mathrm{AKK}$ were given their respective probiotic daily, and HFHC+PBS received phosphate buffered saline. From weeks 12 to 14, the cognitive evaluation took place, at the end of which the animals were sacrificed and the samples were collected. b Body weight across weeks. Two-way ANOVA (Group x Week) was used to assess weight gain. No changes in body weight between groups were found throughout the 14 weeks of the administration of the diet, and significant differences across the weeks were revealed. c Locomotor function evaluation measured on the Rotarodaccelerod test. Bar charts (mean \pm SEM) represent the maximum speed (rpm) of the animals on the rod, compared with the Kruskal-Wallis test. There were no statistically significant differences between groups. d Novel object recognition test. Bar charts (mean \pm SEM) represent the discrimination ratio (d2) between the new object and the one previously observed. Mann-Whitney's $U$ test for independent samples (* 
comparison with zero) and one-way ANOVA followed by Tukey's test (\# comparison of NC, NC+EE, HFHC and $\mathrm{HFHC}+\mathrm{EE}$ d2 value) were used. $\mathrm{NC}$ and $\mathrm{NC}+\mathrm{EE}$ groups were able to recognise the new object; whereas $\mathrm{HFHC}$ was not able to discriminate it, HFHC+EE showed a recovered novel object recognition ability. $\# p<0.05,{ }^{*} p \leq 0.010,{ }^{* \star} p<0.001$. e Spatial working memory test. Bar charts (mean $\pm S E M$ ) represent the average latency on the sample and retention trials. Two-tailed paired $t$-tests (* comparison with its respective sample) and one-way ANOVA followed by Tukey's test (\# comparison between NC, NC+EE and $\mathrm{HFHC}+\mathrm{EE}$ ) were used. $\mathrm{NC}$ and $\mathrm{NC}+\mathrm{EE}$ groups remembered the position of the platform in the retention trial; whereas HFHC was not able to remember it, HFHC + EE displayed recovered spatial working memory. $\# p<0.05,{ }^{\star \star} p \leq 0.010$.

Environmental enrichment cognitive improvement is accompanied by a decrease in brain metabolic activity. The HFHC group showed significantly lower $\mathrm{CCO}$ activity values than the NC group, with less metabolic activity in the infralimbic cortex (IL; $p<0.001)$, cingulated cortex $(\mathrm{Cg}, p<0.001)$, dorsal striatum (dST, $p<0.001$ ), accumbens shell (AcbS; $p<0.001$ ), and perirhinal cortex (PRh; $p=0.002$; Table 1). EE led to a decline in the $\mathrm{CCO}$ levels in the NC $+\mathrm{EE}$ and HFHC + EE groups. The NC + EE group displayed lower CCO levels than the NC group in the prefrontal cortex $(p<0.001)$, dorsal and ventral striatum $(p<0.001)$, thalamus $(p<0.001)$, and CA3 $(p=0.001)$ and DG $(p<0.001)$ hippocampal subregions. In addition, HFHC + EE animals also presented decreased CCO values compared to HFHC animals in the dorsal and ventral striatum ( $p<0.001)$, anterodorsal thalamus (ADT; $p<0.001)$, basolateral amygdala (BLA; $p=0.006)$, dentate gryus (DG; $p<0.001)$, and CA3 $(p=0.001)$. Finally, HFHC + EE showed lower CCO values than NC $+\mathrm{EE}$ in the DG $(p<0.001)$. No differences were found between HFHC + EE and NC+EE in the prefrontal cortex, dorsal and ventral striatum, thalamus, amygdala, and perirhinal cortex. 
Table 1

\section{Brain oxidative metabolism in different groups subjected to NC and HFHC diets and EE.}

The CCO values are expressed as mean \pm SEM. The studied regions included the prefrontal cortex (prelimbic (PrL), infralimbic (IL) and cingulate $(\mathrm{Cg})$ cortex), the dorsal striatum (dST), the ventral striatum (accumbens core (AcbC) and shell (AcbSh)), the thalamus (anteromedial nucleus (AMT), anterodorsal nucleus (ADT), and anteroventral nucleus $(\mathrm{AVT})$ ), the amygdala (central (CeA), basolateral (BLA) and lateral ( $\mathrm{LaA})$ ), the dorsal hippocampus (dentate gyrus (DG), CA1 and CA3 areas), and the perirhinal (PRh) and entorhinal (Ent) cortices. Data were analysed through one-way ANOVA followed by Tukey's test (*\#\&p<0.05; * NC + EE and HFHC vs. NC; \# HFHC + EE vs. HFHC; \& HFHC + EE vs. NC +

$\mathrm{EE})$.

\begin{tabular}{|lllll|}
\hline Region & NC & NC+EE & HFHC & HFHC+EE \\
\hline PrL & $28.526 \pm 1.327$ & $* 21.506 \pm 1.501$ & $21.815 \pm 1.042$ & $* 19.999 \pm 0.862$ \\
\hline IL & $28.153 \pm 1.109$ & $* 21.433 \pm 1.323$ & $* 22.027 \pm 0.939$ & $* 19.634 \pm 0.930$ \\
\hline Cg & $28.846 \pm 1.333$ & $* 22.008 \pm 1.283$ & $* 22.684 \pm 1.164$ & $* 20.495 \pm 0.791$ \\
\hline dST & $28.040 \pm 0.707$ & $* 22.115 \pm 0.622$ & $* 23.078 \pm 0.671$ & $* \# 19.889 \pm 0.784$ \\
\hline AcbC & $35.734 \pm 1.337$ & $* 26.434 \pm 1.192$ & $31.252 \pm 0.903$ & $* \# 22.246 \pm 0.780$ \\
\hline AcbSh & $39.684 \pm 1.055$ & $* 29.814 \pm 1.122$ & $* 34.000 \pm 1.150$ & $* \# 25.962 \pm 0.693$ \\
\hline AMT & $26.087 \pm 1.280$ & $* 16.413 \pm 2.136$ & $22.203 \pm 0.694$ & $* 17.540 \pm 0.824$ \\
\hline ADT & $37.683 \pm 1.684$ & $* 28.188 \pm 1.054$ & $33.995 \pm 1.348$ & $* \# 25.138 \pm 1.207$ \\
\hline AVT & $31.661 \pm 1.702$ & $* 22.988 \pm 1.793$ & $27.684 \pm 1.193$ & $* 22.094 \pm 0.700$ \\
\hline CeA & $23.299 \pm 0.984$ & $19.247 \pm 2.411$ & $22.482 \pm 1.040$ & $* 16.578 \pm 1.151$ \\
\hline BLA & $25.912 \pm 1.338$ & $23.639 \pm 1.334$ & $25.180 \pm 0.988$ & $* \# 19.254 \pm 1.343$ \\
\hline LaA & $20.135 \pm 0.828$ & $20.268 \pm 2.077$ & $20.049 \pm 0.641$ & $14.897 \pm 1.085$ \\
\hline DG & $34.185 \pm 1.729$ & $* 27.210 \pm 0.723$ & $30.501 \pm 0.954$ & $* \# \& 22.593 \pm 0.770$ \\
\hline CA1 & $20.905 \pm 1.154$ & $16.481 \pm 1.709$ & $17.978 \pm 0.805$ & $* 14.541 \pm 1.231$ \\
\hline CA3 & $20.556 \pm 1.127$ & $* 15.018 \pm 1.583$ & $18.396 \pm 0.973$ & $* \# 13.403 \pm 1.072$ \\
\hline PRh & $25.015 \pm 1.240$ & $15.018 \pm 1.583$ & $* 18.891 \pm 0.896$ & $* 17.205 \pm 1.147$ \\
\hline Ent & $21.212 \pm 1.502$ & $17.704 \pm 1.836$ & $17.312 \pm 0.760$ & $16.035 \pm 1.391$ \\
\hline & & & & \\
\hline
\end{tabular}

Environmental enrichment has an effect on microbiota composition and bacterial metabolism. To assess the effect of the environmental enrichment (EE) implementation on the gut microbiota, we studied the gut microbiome profile of the $\mathrm{NC}+\mathrm{EE}$ and $\mathrm{HFHC}+\mathrm{EE}$ groups, by comparing them with the profiles of the control groups (NC and HFHC) and with each other. We first studied how EE affects microbial diversity within the communities by calculating the Chao1 (richness estimator) and Shannon's index (richness and evenness estimator). We confirmed significantly $(p<0.000)$ less bacterial diversity in the HFHC group 
compared to the NC group, as previously described in the characterization of the NASH animal model used [3], and we observed an increase in bacterial diversity in the NC + EE group compared to the NC group $(p<0.01)$. However, EE did not affect the bacterial diversity in the HFHC + EE group, which was significantly lower (also for evenness) than in the NC + EE group $(p<0.000)$ (Fig. 2a).

Next, we assessed the gut microbiota composition in each group of rats. When comparing the NC and HFHC groups, the pattern was substantially different (Additional file 1: Supplementary Figure S1), in agreement with previous studies [3]. The main differences were found in the greater abundance of Lactobacillaceae and Ruminococacceae in the NC groups compared to the HFHC groups, which harboured a greater abundance of Enterobacteriaceae, Bacteroidaceae, or Peptostreptococcaceae. Then, in order to explore how EE could affect the different phylotypes on the microbiota, we applied a linear discriminant analysis effect size (LEfSe) method at the family level to investigate the taxa most likely to explain differences in abundances across the groups. When we compared the four animal groups: NC, $\mathrm{NC}+\mathrm{EE}, \mathrm{HFHC}, \mathrm{HFHC}+\mathrm{EE}$, the results identified statistically significant increased abundance of Micrococcaceae, Christensenellaceae, and Ruminococcaceae in the NC + EE group compared to the rest of the groups, and increased abundance of different families belonging to the Firmicutes phyla and Akkermansiaceae as the most differential microorganisms in the HFHC + EE when compared with the other three groups (Fig. 2b).

To explore the metabolic implications of the microbial differences observed after EE implementation, we analysed the main SCFAs and branched-chain fatty acids (BCFAs) derived from the bacterial metabolism. First, when comparing the HFHC group and the NC group, we confirmed the previous observations [3], where the main SCFAs (acetate, $p<0.01$; propionate, $p<0.05$; and butyrate, $p<0.01$ ) showed statistically significant lower concentrations in the HFHC group. Then, the comparisons of $\mathrm{NC}+\mathrm{EE}$ and $\mathrm{HFHC}+\mathrm{EE}$ with their control groups ( $\mathrm{NC}$ and HFHC, respectively) did not show any significant differences in acetate, propionate, butyrate, or valerate, even though a decreasing tendency was observed after EE. On the other hand, the concentrations of the BCFAs iso-butyric $(p<0.05)$ and iso-valeric (n.s.) were lower in $\mathrm{NC}+\mathrm{EE}$ and $\mathrm{HFHC}+\mathrm{EE}$, compared to $\mathrm{NC}$ and $\mathrm{HFHC}$, respectively (Fig. 2c).

Box-and-whiskers (median and IRQ range) represent comparisons of alpha-diversity of gut microbiota using Chao1 and Shannon indexes among the groups studied, compared using a one-way ANOVA followed by Tukey's test (* comparison with all the groups; \# comparison with HFHC+EE group; \$ comparison with HFHC and HFHC+EE groups). ${ }^{*} \$ \$ p<0.01$. b Gut microbiota composition. Taxonomic cladogram obtained from LEfSe analysis (LDA scores $>2$ and significance of $p<0.05$ as determined by Wilcoxon's signed-rank test) showing bacterial taxa with differential abundance among the groups studied. Red indicates differential abundances in the HFHC group; green indicates differential abundances in the HFHC+EE group; blue indicates differential abundances in the NC group; purple indicates differential abundances in the NC+EE group. c SCFA and BCFAs. Bar charts (mean \pm SEM) represent comparison of the SCFA and BCFA levels ( $\mathrm{mM}$ ) compared using the Kruskal-Wallis test followed by Dunn's analysis ( ${ }^{*}$ comparison with HFHC and HFHC+EE groups; \# comparison with NC+EE group; $\$$ comparison with NC+EE and HFHC+EE groups). ${ }^{*} \# \$ p<0.05$. 
Akkermansia muciniphila, as a probiotic treatment, restores cognition deficits. Slight changes were found in the body weights of the groups throughout the 14 weeks of the administration of the diet because the HFHC + LGG group gained more weight than the NC group $(p=0.038)$; significant differences were observed across weeks $(p<0.001)$, as expected (Fig. 3a). The normal increase in weight was accompanied by the absence of locomotor deficits in all groups ( $p=0.092 ;$ Fig. $3 b)$. On the object recognition test, the experimental groups did not show significant differences between e1 and e2 (NC: $p=$ 0.153; HFHC + PBS: $p=0.565 ; \mathrm{HFHC}+\mathrm{LGG}: p=0.157 ; \mathrm{HFHC}+\mathrm{AKK}: p=0.642$ ) while performing the novel object recognition test. Indeed, the NC group was able to discriminate between a previously encountered object when $\mathrm{d} 1$ and $\mathrm{d} 2$ were statistically higher than zero ( $\mathrm{d} 1: p<0.001 ; \mathrm{d} 2: p<0.001)$. However, the $\mathrm{HFHC}+$ PBS group was not able to discriminate the new object ( $\mathrm{d} 1: p=1.000 ; \mathrm{d} 2: p=1.000)$. After the administration of probiotics (AKK or LGG), the HFHC + LGG group was still unable to recognise the new object (d1: $p=1.000 ; \mathrm{d} 2: p=1.000)$, but the HFHC + AKK group was able to do so (d1: $p=0.010 ; \mathrm{d} 2: p=$ 0.010). When we compared the discrimination ratio between the groups, we found that $\mathrm{NC}$ and $\mathrm{HFHC}+$ AKK showed significantly higher $\mathrm{d} 2$ than HFHC + PBS and HFHC + LGG $(p<0.001)$. These results showed that HFHC + PBS displayed an object recognition impairment that was not recovered by the administration of LGG, but it was reversed by AKK (Fig. 3c).

Finally, when we evaluated the efficacy of probiotic administration on a relevant prefrontal dependent task such as spatial working memory, we found that the HFHC + LGG group was unable to execute the task $(p=0.486)$, as well as the HFHC + PBS group $(p=0.103)$. However, the HFHC + AKK group performed the task ( $p=0.019)$ as correctly as the NC group, and they were able to remember the position of the platform because they showed a statistically significant lower retention latency compared to the sample trials $(p=0.003)$. When we compared the mean latencies in each trial between groups, we observed that, whereas the sample latencies were statistically similar, $(p=0.798)$, the retention latencies differed $(p=$ 0.003) because they were significantly higher in HFHC + PBS and HFHC + LGG than in NC and HFHC + AKK (Fig. 3d). These results highlight the efficacy of $A$. muciniphila CIP107961 as a probiotic treatment to reverse impaired spatial working memory, compared to other probiotics such as L. rhamnosus GG.

Two-way ANOVA (Group x Week) was used to assess weight gain. Slight significant changes in body weight between groups were found throughout the 14 weeks of the administration of the diet because the HFHC+LGG group gained more weight than the NC group; significant differences across the weeks were also revealed. ${ }^{*} p<0.05$. b Locomotor function evaluation measured on Rotarod-accelerod test. Bar charts (mean $\pm S E M$ ) represent the maximum speed (rpm) of the animals on the rod, compared using the KruskalWallis test. There were no statistically significant differences between groups. c Novel object recognition test. Bar charts (mean \pm SEM) represent the discrimination ratio (d2) between the new object and the one previously observed. Mann-Whitney's $U$ test for independent samples (* comparison with zero) and oneway ANOVA followed by Tukey's test (\# comparison of NC, HFHC+PBS, HFHC+LGG and HFHC+AKK d2 value) were used. The NC group was able to recognise the new object; whereas HFHC+PBS and HFHC+LGG were not able to discriminate it, HFHC+AKK showed a recovered novel object recognition ability. $\# p<0.05, \star \star p \leq 0.010, \star \star \star p<0.001$. d Spatial working memory test. Bar charts (mean $\pm S E M$ ) represent the average latency on sample and retention trials. Two-tailed paired t-tests (* comparison with 
its respective sample) and one-way ANOVA followed by Tukey's test (\# comparison between NC, $\mathrm{HFHC}+\mathrm{PBS}, \mathrm{HFHC}+\mathrm{LGG}$, and HFHC+AKK) were used. The NC group remembered the position of the platform on the retention trial; whereas HFHC+PBS and HFHC+LGG were not able to remember it, HFHC+AKK displayed recovered spatial working memory. ${ }^{*} \# p<0.05,{ }^{*} p \leq 0.010$.

Akkermansia muciniphila restores brain metabolic activity to normal. When we explored the brain metabolic activity underlying these cognitive changes, we first found that the HFHC + PBS group showed significantly lower levels of $\mathrm{CCO}$ activity than the NC group in the prefrontal cortex $(p<0.001)$, dorsal and ventral striatum $(p<0.001)$, amygdala nuclei such as CeA $(p=0.004), \operatorname{BLA}(p=0.014)$, hippocampus ( $p \leq$ $0.007)$, and PRh $(p<0.001$; Table 2$)$.

When the animals were treated with A. muciniphila CIP107961, their brain metabolic activity equalled that of the NC group in most of the regions previously affected by HFHC, such as the prefrontal cortex, dorsal striatum, amygdala, hippocampus, and perirhinal cortex. In line with this, HFHC + AKK showed an increased CCO value in the dorsal striatum (dST, $p<0.001$ ), central amygdala (CeA, $p=0.004$ ), and basolateral amygdala (BLA, $p=0.014$ ), compared to HFHC + PBS. However, the HFHC + AKK group also showed lower CCO levels than the NC group in the infralimbic cortex $(\mathrm{IL}, p<0.001)$, ventral striatum $(p<$ $0.001)$, and dentate gyrus (DG, $p<0.001)$.

Regarding L. rhamnosus GG administration, we found that the HFHC + LGG group maintained decreased levels of CCO, compared to the NC group, in the prefrontal cortex $(p<0.001)$, dorsal and ventral striatum $(p<0.001)$, thalamic nuclei such as anteromedial nucleus (AMT, $p=0.016)$, hippocampus $(p \leq 0.007)$, and perirhinal cortex (PRh, $p<0.001)$. More importantly, HFHC + LGG did not display significant differences in CCO levels compared to HFHC + PBS.

When comparing the two probiotic treatments, we observed that the HFHC + AKK group showed statistically significant higher CCO values than the HFHC + LGG group in the prefrontal cortex $(p<0.001)$ and dST $(p<0.001)$. These results point out that the decreased CCO shown by HFHC + PBS cannot be recovered with LGG administration, whereas the opposite effect was found when applying AKK. More importantly, A. muciniphila CIP107961 was able to reverse the HFHC-associated decrease in CCO activity in most of the brain regions previously affected by the HFHC diet. 
Table 2

Brain oxidative metabolism in experimental groups subjected to PBS, LGG and AKK. The CCO values are expressed as mean \pm SEM. The studied regions included the prefrontal cortex (prelimbic (PrL), infralimbic (IL) and cingulate $(\mathrm{Cg})$ cortex), the dorsal striatum (dST), the ventral striatum (accumbens core (AcbC) and shell (AcbSh)), the thalamus (anteromedial nucleus (AMT), anterodorsal nucleus (ADT), and anteroventral nucleus $(\mathrm{AVT})$ ), the amygdala (central (CeA), basolateral (BLA) and lateral ( $\mathrm{LaA})$ ), the dorsal hippocampus (dentate gyrus (DG), CA1 and CA3 areas), and the perirhinal (PRh) and entorhinal (Ent) cortices. Data were analysed through one-way ANOVA followed by Tukey's test (*\#\& $<<0.05$; * HFHC + PBS vs. HFHC + LGG and HFHC + AKK vs. NC; \# HFHC + LGG and $\mathrm{HFHC}+\mathrm{AKK}$ vs. HFHC + PBS; \& HFHC + AKK vs. HFHC + LGG).

\begin{tabular}{|lllll|}
\hline Region & NC & HFHC + PBS & HFHC + LGG & HFHC + AKK \\
\hline PrL & $28.526 \pm 1.327$ & $* 22.244 \pm 1.336$ & $* 20.738 \pm 0.403$ & $\& 25.929 \pm 1.079$ \\
\hline IL & $28.153 \pm 1.109$ & $* 21.897 \pm 0.998$ & $* 19.785 \pm 0.510$ & $* \& 24.277 \pm 1.112$ \\
\hline Cg & $28.846 \pm 1.333$ & $* 22.650 \pm 0.991$ & $* 22.101 \pm 0.545$ & $\& 26.131 \pm 1.077$ \\
\hline dST & $28.040 \pm 0.707$ & $* 22.484 \pm 0.878$ & $* 22.844 \pm 0.473$ & $\# \& 26.125 \pm 0.775$ \\
\hline AcbC & $35.734 \pm 1.337$ & $* 26.777 \pm 0.920$ & $* 24.850 \pm 0.786$ & $* 28.662 \pm 1.185$ \\
\hline AcbSh & $39.684 \pm 1.055$ & $* 30.770 \pm 1.405$ & $* 29.465 \pm 1.298$ & $* 31.602 \pm 1.731$ \\
\hline AMT & $26.087 \pm 1.280$ & $22.187 \pm 0.556$ & $* 21.189 \pm 0.815$ & $23.501 \pm 0.897$ \\
\hline ADT & $37.683 \pm 1.684$ & $35.689 \pm 1.132$ & $34.485 \pm 0.999$ & $37.131 \pm 0.992$ \\
\hline AVT & $31.661 \pm 1.702$ & $27.654 \pm 1.206$ & $27.254 \pm 0.856$ & $29.324 \pm 0.708$ \\
\hline CeA & $23.299 \pm 0.984$ & $* 18.874 \pm 0.849$ & $20.607 \pm 0.456$ & $\# 22.133 \pm 0.827$ \\
\hline BLA & $25.912 \pm 1.338$ & $* 21.297 \pm 1.131$ & $22.484 \pm 0.400$ & $* \# 25.123 \pm 0.628$ \\
\hline LaA & $20.135 \pm 0.828$ & $18.128 \pm 0.688$ & $19.400 \pm 0.342$ & $20.002 \pm 0.889$ \\
\hline DG & $34.185 \pm 1.729$ & $* 28.133 \pm 1.170$ & $* 25.733 \pm 0.726$ & $* 29.408 \pm 0.964$ \\
\hline CA1 & $20.905 \pm 1.154$ & $* 17.692 \pm 0.686$ & $* 16.905 \pm 0.442$ & $17.929 \pm 0.695$ \\
\hline CA3 & $20.556 \pm 1.127$ & $* 16.890 \pm 0.274$ & $* 16.615 \pm 0.237$ & $17.787 \pm 0.669$ \\
\hline PRh & $25.015 \pm 1.240$ & $* 20.272 \pm 0.405$ & $* 18.797 \pm 0.554$ & $19.835 \pm 0.510$ \\
\hline Ent & $21.212 \pm 1.502$ & $17.752 \pm 0.405$ & $17.348 \pm 0.368$ & $18.844 \pm 1.051$ \\
\hline
\end{tabular}

Probiotics do not induce major rearrangements in the faecal microbiota. To assess the effect of two different probiotics on the NASH-associated cognitive disturbances, we first analysed the effect of the administration of the strains by oral gavage on the gut microbiota in the gavage feeding groups (HFHC + AKK, HFHC + LGG, and HFHC + PBS [control group]), also including the NC group (no gavage) as external control. Miseq sequencing produced an average of $\sim 63,000$ filtered partial sequences per sample, and it showed the biggest differences at the diversity and compositional levels between the NC group and the other three NASH groups (Fig. 4a, Additional file 1: Supplementary Figure S2a), confirming the strong 
effect of diet (control vs. HFHC diet). The analyses of the SCFAs followed the same trend (Additional file 1: Supplementary Figure S2b). These results are in concordance with our previous observations in the $\mathrm{NASH}$ [3] animal model. Thus, by focusing more on the specific effect of the two probiotics on the gut microbiota, we observed that the administration of both bacteria led to a unique change in the microbial composition at the phylum level, by significantly decreasing Bacteroidetes, compared to placebo administration (PBS) ( $p=0.016 \mathrm{HFHC}+\mathrm{LGG}$ vs. HFHC + PBS; $p=0.018 \mathrm{HFHC}+\mathrm{AKK}$ vs. HFHC + PBS). At lower taxonomical levels, applying a linear discriminant analysis effect size (LEfSe) method, we observed that only a few families and genus suffered differential changes in their abundance depending on the probiotic administration. LGG produces, among others, a statistically significant increase in the relative abundance of Christensenellaceae, Ruminococcaceae, Peptococcaceae, and Lactobacillus (Fig. 4b). In the HFHC + AKK group, we observed higher abundance mainly in Faecalibacterium, Prevotella 9, and the Ruminococcus UCG005 group (Fig. 4b). Changes in the concentration of the Lactobacillus and Akkermansia genus were validated by qPCR, confirming higher levels of the Lactobacillus genus in the $\mathrm{HFHC}+$ LGG group and no differences in the Akkermansia genus across the groups (Fig. 4c). On the other hand, the Chao1 index showed statistically higher alpha-diversity in the HFHC + AKK group when compared with the HFHC + PBS group $(p<0.05)$; however, the Shannon index did not show any significant differences among the groups (Fig. 4d). These results indicate that probiotics can affect microbial diversity, but they did not induce major rearrangements of the faecal microbiota.

Average relative abundance of gut microbiota at the family level from NC, HFHC+PBS, HFHC+LGG, and $\mathrm{HFHC}+\mathrm{AKK}$ groups. Bacterial taxa representing less than $0.5 \%$ of the total abundance are included in Others. $\mathbf{b}$ Gut microbiota differences. Results of LEfSe analysis (LDA scores $>2$ and significance of $p<0.05$ as determined by Wilcoxon's signed-rank test) showing significantly different taxa among the $\mathrm{HFHC}+\mathrm{PBS}, \mathrm{HFHC}+\mathrm{LGG}, \mathrm{HFHC}+\mathrm{AKK}$ groups. Red indicates differential abundance in the HFHC+AKK group; green indicates differential abundance in the HFHC+LGG group; blue indicates differential abundance in the NC+PBS group. Bacterial taxa representing less than $0.5 \%$ of the total abundance are included in Others. c qPCR concentration. Bar charts (mean \pm SEM) represent comparison of Lactobacillus and Akkermansia genus concentration analysed by qPCR ( $\log _{10}$ cells $/ \mathrm{g}$ faeces), compared using the Kruskal-Wallis test followed by Dunn's analysis ( ${ }^{*} p<0.05$, comparison with all the groups). $\mathbf{d}$ Bacterial diversity. Box-and-whiskers (median and IRQ range) represent comparison of alpha-diversity of gut microbiota using Chao1 and Shannon indexes among groups, compared using a one-way ANOVA followed by Tukey's test ( ${ }^{*} p<0.05$, comparison with HFHC+PBS group).

Finally, the levels of the main SCFAs did not show differences between the probiotic groups and the $\mathrm{HFHC}+\mathrm{PBS}$ group, with the only exception of acetate, which was lower in the probiotic groups $(p<0.01$, $\mathrm{HFHC}+\mathrm{LGG}$ vs. HFHC + PBS; $p<0.05, \mathrm{HFHC}+$ AKK vs. HFHC + PBS) (Additional file 1: Supplementary Figure $\mathrm{S} 2 \mathrm{c})$.

Cognitive recovery is equally improved by environmental enrichment and Akkermansia muciniphila. No locomotor dysfunction has been found in any experimental group ( $p=0.310 ;$ Fig. 5a). Both treatments, environmental enrichment and the administration of A. muciniphila CIP107961, were able to improve 
novel object recognition in HFHC animals. When we compared the discrimination ratio (d2) between $\mathrm{HFHC}+\mathrm{PBS}, \mathrm{HFHC}+\mathrm{EE}$, and $\mathrm{HFHC}+\mathrm{AKK}$ to observe if there was a differential effect, we found that $\mathrm{HFHC}+\mathrm{EE}$ and $\mathrm{HFHC}+\mathrm{AKK}$ presented a higher $\mathrm{d} 2$ value than HFHC + PBS $(p=0.013)$, but the $\mathrm{d} 2$ value did not differ significantly between the HFHC + EE and HFHC + AKK groups. Thus, environmental enrichment and AKK administration were equally successful in reversing the novel object recognition impairment caused by the HFHC diet (Fig. 5b).

Regarding spatial working memory, we previously showed that EE and AKK improved the performance of $\mathrm{HFHC}$ animals. When comparing the mean latencies of $\mathrm{HFHC}+\mathrm{PBS}, \mathrm{HFHC}+\mathrm{EE}$, and $\mathrm{HFHC}+\mathrm{AKK}$, we observed that, whereas there were no statistically significant differences in the sample trials $(p=0.647)$, both HFHC + EE and HFHC + AKK showed lower retention latencies than HFHC + PBS $(p=0.004)$. However, retention latencies between $\mathrm{HFHC}+\mathrm{EE}$ and $\mathrm{HFHC}+\mathrm{AKK}$ were not significantly different. Thus, $\mathrm{EE}$ and AKK administration were both equally effective in restoring spatial working memory in the HFHC condition (Fig. 5c).

Bar charts (mean \pm SEM) represent the maximum speed (rpm) of the animals on the rod, compared using the Kruskal-Wallis test. There were no statistically significant differences between groups. b Novel object recognition test. Bar charts (mean \pm SEM) represent the discrimination ratio (d2) between the new object and the one previously observed. Mann-Whitney's Utest for independent samples (* comparison with zero) and the Kruskal-Wallis test followed by Tukey's analysis (\# comparison of HFHC+PBS, HFHC+EE and $\mathrm{HFHC}+\mathrm{AKK}$ for $\mathrm{d} 2$ value) were used. $\mathrm{HFHC}+\mathrm{EE}$ and $\mathrm{HFHC}+\mathrm{AKK}$ showed improved recognition of a novel object in comparison with HFHC+PBS animals, but no differences were found between them. $\# p<0.05,{ }^{\star *} p \leq 0.010$. c Spatial working memory test. Bar charts (mean $\left.\pm S E M\right)$ represent the average latency on the sample and retention trials. Two-tailed paired $t$-tests (* comparison with its respective sample) and one-way ANOVA followed by Tukey's test (\# comparison between HFHC+PBS, HFHC+EE and $\mathrm{HFHC}+\mathrm{AKK}$ ) were used. $\mathrm{HFHC}+\mathrm{EE}$ and $\mathrm{HFHC}+\mathrm{AKK}$ displayed improved performance on the spatial working memory task, in comparison with HFHC+PBS, but no differences were found between them. ${ }^{*} \# p<0.05,{ }^{* *} p \leq 0.010$.

\section{Environmental enrichment and Akkermansia muciniphila have differential effects on brain metabolic} activity. Next, we compared CCO values in the HFHC + PBS, HFHC + EE, and HFHC + AKK groups in order to discover whether there was a differential treatment effect on brain metabolism. We first observed that $\mathrm{HFHC}+\mathrm{EE}$ displayed lower CCO levels than HFHC + PBS in the accumbens core (AcbC, $p<0.001)$, thalamus $(p<0.001)$, lateral amygdala ( $\operatorname{LaA}, p=0.002), \mathrm{DG}(p<0.001)$, and CA3 $(p=0.001)$. However, the opposite results were found in the HFHC + AKK group, where the CCO values were higher

than in the HFHC + PBS group in the cingulate cortex $(\mathrm{Cg}, p=0.002)$, dST $(p<0.001)$, and $\operatorname{BLA}(p=0.003)$.

Second, we found that HFHC + AKK showed higher CCO levels than HFHC + EE in the prefrontal cortex ( $p$ $\leq 0.014)$, dorsal and ventral striatum $(p \leq 0.024)$, thalamus $(p<0.001)$, amygdala $(p \leq 0.003)$, hippocampus ( $p \leq 0.026)$, and PRh $(p=0.004)$ (Table 3$)$. Thus, these results highlight that even if 
environmental enrichment and A. muciniphila CIP107961 lead to similar cognitive improvement, the brain metabolic modifications underlying this amelioration are different.

Table 3

Brain oxidative metabolism in HFHC groups subjected to PBS, AKK and EE. The CCO values are expressed as mean \pm SEM. The studied regions included the prefrontal cortex (prelimbic (PrL), infralimbic (IL) and cingulate $(\mathrm{Cg})$ cortex), the dorsal striatum (dST), the ventral striatum (accumbens core (AcbC) and shell (AcbSh)), the thalamus

(anteromedial nucleus (AMT), anterodorsal nucleus (ADT), and anteroventral nucleus (AVT)), the amygdala (central (CeA), basolateral (BLA) and lateral ( $(\mathrm{AA} A)$ ), the dorsal hippocampus (dentate

gyrus (DG), CA1 and CA3 areas), and the perirhinal (PRh) and

entorhinal (Ent) cortices. Data were analysed through one-way ANOVA followed by Tukey's test $(* \# p<0.05 ; * \mathrm{HFHC}+\mathrm{EE}$ and $\mathrm{HFHC}+$ AKK vs. HFHC + PBS; \# HFHC + AKK vs. HFHC + EE).

\begin{tabular}{|llll|}
\hline Region & HFHC + PBS & HFHC + EE & HFHC + AKK \\
\hline PrL & $22.244 \pm 1.336$ & $19.999 \pm 0.862$ & $\# 25.929 \pm 1.079$ \\
\hline IL & $21.897 \pm 0.998$ & $19.634 \pm 0.930$ & $\# 24.277 \pm 1.112$ \\
\hline Cg & $22.650 \pm 0.991$ & $20.495 \pm 0.791$ & $* \# 26.131 \pm 1.077$ \\
\hline dST & $22.484 \pm 0.878$ & $19.889 \pm 0.784$ & $* \# 26.125 \pm 0.775$ \\
\hline AcbC & $26.777 \pm 0.920$ & $* 22.246 \pm 0.780$ & $\# 28.662 \pm 1.185$ \\
\hline AcbSh & $30.770 \pm 1.405$ & $25.962 \pm 0.693$ & $\# 31.602 \pm 1.731$ \\
\hline AMT & $22.187 \pm 0.556$ & $* 17.540 \pm 0.824$ & $\# 23.501 \pm 0.897$ \\
\hline ADT & $35.689 \pm 1.132$ & $* 25.138 \pm 1.207$ & $\# 37.131 \pm 0.992$ \\
\hline AVT & $27.654 \pm 1.206$ & $* 22.094 \pm 0.700$ & $\# 29.324 \pm 0.708$ \\
\hline CeA & $18.874 \pm 0.849$ & $16.578 \pm 1.151$ & $\# 22.133 \pm 0.827$ \\
\hline BLA & $21.297 \pm 1.131$ & $19.254 \pm 1.343$ & $* \# 25.123 \pm 0.628$ \\
\hline LaA & $18.128 \pm 0.688$ & $* 14.897 \pm 1.085$ & $\# 20.002 \pm 0.889$ \\
\hline DG & $28.133 \pm 1.170$ & $* 22.593 \pm 0.770$ & $\# 29.408 \pm 0.964$ \\
\hline CA1 & $17.692 \pm 0.686$ & $14.541 \pm 1.231$ & $\# 17.929 \pm 0.695$ \\
\hline CA3 & $16.890 \pm 0.274$ & $* 13.403 \pm 1.072$ & $\# 17.787 \pm 0.669$ \\
\hline PRh & $20.272 \pm 0.405$ & $17.205 \pm 1.147$ & $\# 19.835 \pm 0.510$ \\
\hline Ent & $17.752 \pm 0.405$ & $16.035 \pm 1.391$ & $18.844 \pm 1.051$ \\
\hline
\end{tabular}

Environmental enrichment and Akkermansia muciniphila differentially influence microbiota. Our previous results showed that both environmental enrichment implementation and administration of $A$. muciniphila CIP107961 improved novel object recognition, spatial working memory performance, and changes in the 
brain metabolism in HFHC animals. Both interventions also produced slight changes in the microbial composition compared to their control groups, as we explained above. When comparing the microbial composition of HFHC + EE and HFHC + AKK, noteworthy differences were found (Fig. 6); however, this could be explained by initial background differences in the faecal microbiota composition of the litters before treatment $(\mathrm{HFHC}+\mathrm{EE}$ and $\mathrm{HFHC}+\mathrm{AKK})(76.29 \%-68.65 \%$ of the relative abundance of Firmicutes, respectively; $13.56 \%-17.92 \%$ of Proteobacteria; $9.21 \%-9.33 \%$ of Bacteroidetes; $0.40 \%-3.78 \%$ of Verrucomicrobia; $0.37 \%-0.33 \%$ of Actinobacteria). On the other hand, when comparing HFHC + AKK vs. $\mathrm{HFHC}+\mathrm{PBS}$, we observed that the probiotic did not induce a major community-wide compositional change, compared to the corresponding control group (HFHC + PBS). Only some previously mentioned changes were observed in the HFHC + AKK group.

Average relative abundance of prevalent microbiota at the family level from $\mathrm{HFHC}+\mathrm{EE}, \mathrm{HFHC}+\mathrm{PBS}$, $\mathrm{HFHC}+\mathrm{AKK}$ groups. Bacterial taxa representing less than $0.5 \%$ of the total abundance are included in Others.

Regarding the levels of the main SCFAs, only propionate showed different concentrations between HFHC + EE and the HFHC + AKK and HFHC + PBS groups; and in the BCFA, iso-butyrate reached higher concentrations in the HFHC + AKK group, compared to the placebo group and the HFHC + EE group (Additional file 1: Supplementary Figure S3).

\section{Discussion}

In this study, we described cognitive, brain metabolism, and microbiota alterations associated with highfat and high-cholesterol consumption. In addition, we clearly showed that environmental enrichment and A. muciniphila CIP107961 restore cognitive dysfunction. Furthermore, we revealed that cognitive improvement was due to differential effects of environmental enrichment and this strain of $A$. muciniphila on brain metabolism and gut microbiota. Finally, we discovered that restored cognitive function was associated with the administration of $A$. muciniphila CIP107961, but not L. rhamnosus GG, which may be clinically relevant when selecting probiotics for treating HFHC-derived pathologies.

Environmental enrichment (EE) has been described as motor and sensorial stimulation that is able to modulate the physical and cognitive status [18]. Moreover, EE has been found to have brain repairing therapeutic effects in several neuropsychiatric diseases [17-19]. Our results revealed that the HFHC group presented cognitive deficits. Specifically, novel object recognition impairment was restored by EE, in accordance with de Souza et al. [41]. Our data extend these findings demonstrating that spatial working memory alterations, which we have previously described [3], were also improved by the EE. The EE's effect on the HFHC group was accompanied by decreased levels of brain metabolism in the prefrontal cortex, striatum, and perirhinal cortex. Moreover, other brain regions such as the thalamus, the amygdala, and the hippocampus showed the same pattern. The prefrontal cortex sends projections to the perirhinal and entorhinal cortices [42] and is in reciprocal connection with the striatum [43] and the thalamus [44]. This network supports spatial working memory and novel object recognition abilities [34, 42, 44, 45]. Therefore, 
any improvement in this network will be reflected in an improvement on both behavioural tasks, as we demonstrated.

One additional finding is the decrease in brain metabolic levels when introducing the $E E$, which was clearly observed not only in the HFHC + EE group, but also in the NC + EE group. The NC + EE group displayed lower $\mathrm{CCO}$ values than the NC group in the prefrontal cortex, striatum, thalamus, and hippocampus, whereas HFHC + EE showed lower levels than HFHC in the striatum, thalamus, amygdala, and hippocampus. These results are in line with previous data by Tong et al. [46] showing that exercise decreased the hippocampal expression of genes involved in mitochondrial metabolic processes, including cytochrome c-oxidase. In fact, physical activity and EE have been found to regulate metabolic and redox activity, resulting in a beneficial modulation of brain function [47]. Likewise, our results support the overall effect of EE on brain metabolism through the reduction in metabolic levels in both healthy and HFHC conditions.

When we assessed the gut microbiota composition of the HFHC and NC groups, we confirmed previous observations in the same HFHC animal model [3], such as lower diversity and important changes at the compositional level. At the phylum level, the HFHC group showed higher levels of Proteobacteria and lower levels of Firmicutes, alterations that were translated into lower taxonomical levels. Changes in the gastrointestinal microbial population correlate with specific patterns of faecal metabolites [48], and we observed that the faecal metabolite profile also showed alterations with respect to the control group, with lower concentrations of the main SCFAs in the faecal water of the HFHC group. Physical exercise appears to be able to modify microbiota [49]. In our experimental model, the EE did not induce a significant community-wide compositional change when applied to the NC group, although the bacterial diversity richness increased in the NC + EE group. However, EE induced more modifications at the taxonomical level in the HFHC group, decreasing Proteobacteria and increasing Firmicutes. These changes were accompanied by decreased iso-butyrate levels, but without any change in the bacterial diversity. This is consistent with the findings of Allen et al. [50], who showed that the effect of EE was dependent on the health status of their experimental groups because obese subjects showed a different response to exercise from that of healthy controls in terms of gut microbiota. In our case, EE did not affect the gut microbiota of the healthy control group; however, the HFHC + EE displayed a significant modification of the microbiota composition, illustrating the complex association between microbiota and health.

Gut microbiota changes provoke a dysregulation of the enteric nervous system, which leads to a breakage of the gut-brain axis and ultimately to neural deficits $[10,51]$. In addition, gut dysbiosis has been associated with mitochondrial dysfunction through the delivery of metabolites that can affect reactive oxygen species and ATP production [51], suggesting a potential mechanism for neural damage. The NC, NC + EE, and HFHC + EE groups did not show the cognitive impairment displayed by the HFHC group; they manifested a better capability for novel object recognition and spatial working memory. Hence, these results demonstrated that $\mathrm{EE}$ is able to target the gut-brain axis, leading to the modulation of brain oxidative metabolism, which has an impact on cognitive amelioration. 
In a previous pilot study, we found that these gut microbiota changes in the HFHC + EE group were reflected in a significant increase in Akkermansia levels compared to the HFHC group. Moreover, the comparison of the $\mathrm{NC}$ and $\mathrm{HFHC}$ groups indicated a reduction in the relative proportions of lactobacilli in the HFHC group. These two observations prompted us to evaluate the impact on cognition of the administration of two strains of the cited microbial groups, L. rhamnosus $G G$ and the potentially novel probiotic $A$. muciniphila CIP107961, for which beneficial effects have been recently reported [23-25].

The administration of LGG was not effective in ameliorating the cognitive dysfunction in the HFHC group, showing similar results to those found in the control group (HFHC + PBS). These results are in line with a previous study by Kelly et al. [52] in healthy humans, where LGG was not able to improve cognitive function, measured as visuospatial memory performance, attention switching, rapid visual information processing, and emotion recognition. At the compositional level, the gut microbiota of the HFHC + LGG group suffered slight modifications with regard to the placebo group (HFHC + PBS), with a considerable increase in lactobacilli and a lower concentration of acetate in the faecal water. In contrast to the results observed with LGG, the HFHC animals receiving A. muciniphila CIP107961 displayed correct performance on novel object recognition and spatial working memory, which means that this microorganism is effective in reversing HFHC-associated cognitive defects. Previous studies had found that $A$. muciniphila was effective in metabolic disorders derived from HFHC diets by reversing endotoxemia $[53,54]$. In this way, it has been observed that $A$. muciniphila can also lower plasma LPS levels [26] through the reinforcement of the gut barrier. Moreover, several studies have shown that the administration of this microorganism, either dead or alive in animal models, reduced levels of different hepatic enzymes considered to be markers of inflammation and hepatic steatosis $[26,54,55]$. These enzymes have been found to be increased in our HFHC model [56], along with the presence of hepatic steatosis [3]. In accordance with these physiological benefits, for the first time, our results show a potential role of $A$. muciniphila in improving cognitive function.

We found that $A$. muciniphila CIP107961 not only restored metabolic levels in the amygdala and the dorsal striatum and increased the CCO values compared to HFHC + LGG in this latter area, but higher CCO values were also found in the prefrontal cortex. Moreover, the group treated with A. muciniphila CIP107961 displayed similar values to the NC group in regions previously affected by HFHC, such as the prefrontal cortex, striatum, hippocampus, and perirhinal cortex. When we addressed the L. rhamnosus GG administration, no changes were found in the $\mathrm{CCO}$ values in any of the measured regions compared to $\mathrm{HFHC}+\mathrm{PBS}$, which is consistent with the inability of this group to improve their cognitive function. Previous studies have suggested that $A$. muciniphila is associated with endocannabinoid regulation because its administration led to increased intestinal levels of endocannabinoids [53], and, in turn, the blockage of endocannabinoid receptor CB1 increased levels of this microorganism [57]. The endocannabinoid system can be modulated along the gut-brain axis through vagal control [58], and CB1 receptors, which are densely located in the striatum and hippocampus [59,60], have been suggested to be present in the mitochondrial membrane, so that they are able to control cellular respiration and energy production [61]. Therefore, this aforementioned endocannabinoid regulation of $A$. muciniphila in the 
intestine could be extending its effects along the gut-brain-axis, ultimately manifested as mitochondrial activity upregulation, which could be causing the cognitive improvement.

An interesting finding of our study is the decreased CCO levels when comparing the HFHC + PBS and NC groups in the prefrontal, hippocampus, amygdala and perirhinal cortices. These results could be reflecting a stress response $[62,63]$ potentially induced by gavage administration [64]. The gut microbiota study after $A$. muciniphila CIP107961 administration did not show major rearrangements, with only slight modifications and a modest increase in bacterial diversity when compared with the microbiota of animals receiving PBS or LGG. This suggests that major microbiota changes are not involved in the observed effects, which is in line with previous studies carried out in other animal models $[24,54]$ or in humans [26] that reported beneficial effects of this microorganisms without observing major changes in the gut microbiota composition. However, it is important to note that more in-depth studies may be needed to fully elucidate this issue because in this study we rely on 16S rRNA gene-based microbiota profiling, and, therefore, we do not have a complete overview of the total intestinal metagenome.

We finally wanted to compare the two successful treatments, environmental enrichment and $A$. muciniphila CIP107961 administration, in order to elucidate their differential effects along the gut-brain axis. We first showed that each treatment produces an opposite effect on brain metabolism; whereas EE caused an overall brain metabolic decrease, AKK restored the activity. Second, in a similar manner, HFHC $+\mathrm{EE}$ and HFHC + AKK animals showed clear differences in their microbiota patterns. Whereas the HFHC + AKK group showed only minor differences with regard to the corresponding experimental control group (HFHC + PBS), the differences with the HFHC + EE group were larger, including higher levels of Bacteroidetes and Proteobacteria at the phylum level. However, as previously discussed, the existence of differences in the basal microbiota between these two groups keeps us from drawing firm conclusions. Indeed, these differences suggest that the observed effects of both EE and A. muciniphila administration on the reversion of NASH-associated cognitive impairments are independent from a defined overall microbiota composition. In this regard, some studies have identified specific $A$. muciniphila proteins, such as the membrane protein Amuc_1100, as being able to mediate some of these beneficial effects [55].

Finally, we want to point out the observed changes in the SCFAs and their metabolites, which showed a decrease in iso-butyrate and acetate when the animals were exposed to environmental enrichment or treated with probiotics, respectively. In this regard, some microorganisms, such as bacteroides or Clostridia members, are able to produce BCFAs from the proteolytic metabolism of branched-chain amino acids. The higher levels of Bacteroidaceae or Peptostreptococcaceae observed in the HFHC group could be related to the higher levels of BCFAs observed, as found in other diseases related to the gut-brain axis, such as obesity [65], Rett syndrome [66], or anorexia nervosa [67]. On the other hand, there is evidence in mice that acetate can alter the levels of glutamate, glutamine, and GABA [68], and some studies have shown that SCFAs and their metabolites can stimulate vagus nerve signaling $[69,70]$. Moreover, other studies have correlated changes in SCFAs and iso-butyrate with positive effects on behaviour through the microbiota-gut-brain axis [8]. Hence, these results could be highlighting the contribution of SCFAs and BCFAs to NASH-cognitive improvement. 


\section{Conclusions}

In conclusion, the microbiota and cognition are intimately connected through the gut-brain axis, and in $\mathrm{HFHC}$ pathologies they can be influenced by environmental enrichment and $A$. muciniphila CIP107961 administration. Cognitive improvement was accompanied by changes in brain metabolic activity and gut microbial composition analysis, pointing to specific microbiota targets for intervention in diet-induced pathologies. However, some mechanisms other than major changes in microbiota composition and the combined effect of environmental enrichment and A. muciniphila administration, which we identified in this study, may also be biologically relevant and will need to be investigated in future studies due to their relative contributions to the selection of effective treatments for patients.

\section{Declarations}

\section{Ethics approval}

The procedures and manipulation of the animals used in this study were carried out according to the Directive (2010/63/EU), Royal Decree 53/2013 of the Ministry of the Presidency related to the protection of animals used for experimentation and other scientific purposes.

\section{Consent for publication}

Not applicable

\section{Availability of data and materials}

The datasets generated during and analysed during the current study are available from the corresponding author on reasonable request.

\section{Competing interests}

The authors declare that they have no competing interests.

\section{Funding}

This work was supported by the Ministry of Science, Innovation and Universities (PSI2017-83893-R to JLA) and the Ministry of Economy and Business (PSI2015-73111-EXP to JLA, PSI2017-90806-REDT to JLA and AGL2017-83653R to MG) (Spain). S.A was the recipient of a postdoctoral Juan de la Cierva Contract (Ministry of Science, Innovation and Universities, Ref. IJCI-2017-32156). The funders had no role in the study design or data collection. 


\section{Author contributions}

S.A., J.A., M.G. and N.A. conceived the concept and designed the experiments. S.H., S.A., M.G. and N.A. performed the experiments and analysed the results. All authors wrote and edited the manuscript.

\section{Acknowledgements.}

We would like to thank the Alzheimer's Research UK King's College London Network Centre for their technical and human support.

\section{References}

1. Diehl AM, Day C. Cause, pathogenesis, and treatment of nonalcoholic steatohepatitis. N Engl J Med. 2017;377:2063-72.

2. Wong VWS, Chitturi S, Wong GLH, Yu J, Chan HLY, Farrell GC. Pathogenesis and novel treatment options for non-alcoholic steatohepatitis. Lancet Gastroenterol Hepatol. 2016;1:56-67.

3. Higarza SG, Arboleya S, Gueimonde M, Go E, Arias JL, Arias N. Neurobehavioral dysfunction in nonalcoholic steatohepatitis is associated with hyperammonemia, gut dysbiosis, and metabolic and functional brain regional deficits. PLoS One. 2019;14:e0223019.

4. Christensen CU, Glavind E, Thomsen KL, Kim YO, Heebøll S, Schuppan D, et al. Niemann-Pick type C2 protein supplementation in experimental nonalcoholic fatty liver disease. PLoS One. 2018;13:e0192728.

5. Turnbaugh PJ, Hamady M, Yatsunenko T, Cantarel BL, Duncan A, Ley RE, et al. A core gut microbiome in obese and lean twins. Nature. 2009;457:480-4.

6. Dalby MJ, Ross AW, Walker AW, Morgan PJ. Dietary uncoupling of gut microbiota and energy harvesting from obesity and glucose tolerance in mice. Cell Rep. 2017;21:1521-33.

7. Hoyles L, Fernández-Real JM, Federici M, Serino M, Abbott J, Charpentier J, et al. Molecular phenomics and metagenomics of hepatic steatosis in non-diabetic obese women. Nat Med. 2018;24:1070-80.

8. Golubeva A V., Joyce SA, Moloney G, Burokas A, Sherwin E, Arboleya S, et al. Microbiota-related changes in bile acid \& tryptophan metabolism are associated with gastrointestinal dysfunction in a mouse model of autism. EBioMedicine. 2017;24:166-78.

9. Dinan TG, Cryan JF. Brain-gut-microbiota axis and mental health. Psychosom Med. 2017;79:920-6.

10. Cryan JF, Dinan TG. Mind-altering microorganisms: the impact of the gut microbiota on brain and behaviour. Nat Rev Neurosci. 2012;13:701-12.

11. Moris G, Arboleya S, Mancabelli L, Milan C, Ventura M, Re CGDL, et al. Fecal microbiota profile in a group of myasthenia gravis patients. Sci Rep. 2018;8:14384. 
12. Strati F, Cavalieri D, Albanese D, De Felice C, Donati C, Hayek J, et al. New evidences on the altered gut microbiota in autism spectrum disorders. Microbiome. 2017;5:24.

13. Clarke SF, Murphy EF, O’Sullivan O, Lucey AJ, Humphreys M, Hogan A, et al. Exercise and associated dietary extremes impact on gut microbial diversity. Gut. 2014;63:1913-20.

14. Barton W, Penney NC, Cronin O, Garcia-Perez I, Molloy MG, Holmes E, et al. The microbiome of professional athletes differs from that of more sedentary subjects in composition and particularly at the functional metabolic level. Gut. 2018;67:625-33.

15. Mancini A, Campagna F, Amodio P, Tuohy KM. Gut: liver: brain axis: the microbial challenge in the hepatic encephalopathy. Food Funct. 2018;9:1373-88.

16. Erickson KI, Voss MW, Prakash RS, Basak C, Szabo A, Chaddock L, et al. Exercise training increases size of hippocampus and improves memory. Proc Natl Acad Sci U S A. 2011;108:3017-22.

17. Hannan AJ. Environmental enrichment and brain repair: Harnessing the therapeutic effects of cognitive stimulation and physical activity to enhance experience-dependent plasticity. Neuropathol Appl Neurobiol. 2014;40:13-25.

18. Pang TYC, Hannan AJ. Enhancement of cognitive function in models of brain disease through environmental enrichment and physical activity. Neuropharmacology. 2013;64:515-28.

19. Fischer A. Environmental enrichment as a method to improve cognitive function. What can we learn from animal models? Neuroimage. 2016;131:42-7.

20. Diamond MC. Response of the brain to enrichment. An Acad Bras Cienc. 2001;73:210-20.

21. Segers ME, Lebeer S. Towards a better understanding of Lactobacillus rhamnosus GG - host interactions. Microb Cell Fact. 2014;13 Suppl 1:S7.

22. Ivanovic N, Minic R, Djuricic I, Radojevic Skodric S, Zivkovic I, Sobajic S, et al. Active Lactobacillus rhamnosus LA68 or Lactobacillus plantarum WCFS1 administration positively influences liver fatty acid composition in mice on a HFD regime. Food Funct. 2016;7:2840-8.

23. Cani PD, de Vos WM. Next-generation beneficial microbes: the case of Akkermansia muciniphila. Front Microbiol. 2017;8:1765.

24. Bárcena C, Valdés-Mas R, Mayoral P, Garabaya C, Durand S, Rodríguez F, et al. Healthspan and lifespan extension by fecal microbiota transplantation in progeroid mice. Nat Med. 2019.

25. Blacher E, Bashiardes S, Shapiro H, Rothschild D, Mor U, Dori-Bachash M, et al. Potential roles of gut microbiome and metabolites in modulating ALS in mice. Nature. 2019;572:474-80.

26. Depommier C, Everard A, Druart C, Plovier H, Van Hul M, Vieira-Silva S, et al. Supplementation with Akkermansia muciniphila in overweight and obese human volunteers: a proof-of-concept exploratory study. Nat Med. 2019;25:1096-103.

27. Arboleya S, Binetti A, Salazar N, Ferna N, Solís G, Hernández-Barranco A, et al. Establishment and development of intestinal microbiota in preterm neonates. FEMS Microbiol Ecol. 2012;79:763-72.

28. Milani C, Hevia A, Foroni E, Duranti S, Turroni F, Gueimonde M, et al. Assessing the fecal microbiota: an optimized ion torrent 16S rRNA gene-based analysis protocol. PLoS One. 2013;8:e68739. 
29. Nogacka A, Salazar N, Suárez M, Milani C, Arboleya S, Solís G, et al. Impact of intrapartum antimicrobial prophylaxis upon the intestinal microbiota and the prevalence of antibiotic resistance genes in vaginally delivered full-term neonates. Microbiome. 2017;5:93.

30. Edgar RC. Search and clustering orders of magnitude faster than BLAST. Bioinformatics. 2010;26:2460-1.

31. Quast C, Pruesse E, Yilmaz P, Gerken J, Schweer T, Glo FO, et al. The SILVA ribosomal RNA gene database project: improved data processing and web-based tools. Nucleic Acids Res. 2013;41:D590596.

32. Valdés L, Salazar N, González S, Arboleya S, Ríos-Covián D, Genovés S, et al. Selection of potential probiotic bifidobacteria and prebiotics for elderly by using in vitro faecal batch cultures. Eur Food Res Technol. 2017;243:157-65.

33. Jones BJ, Roberts DJ. The quantitative measurement of motor inco-ordination in naive mice using an accelerating rotarod. J Pharm Pharmacol. 1968;20:302-4.

34. Mendez M, Arias N, Uceda S, Arias JL. c-Fos expression correlates with performance on novel object and novel place recognition tests. Brain Res Bull. 2015;117:16-23.

35. Morris R. Developments of a water-maze procedure for studying spatial learning in the rat. J Neurosci Methods. 1984;11:47-60.

36. Méndez M, Méndez-López M, López L, Aller MA, Arias J, Arias JL. Working memory impairment and reduced hippocampal and prefrontal cortex c-Fos expression in a rat model of cirrhosis. Physiol Behav. 2008;95:302-7.

37. Méndez-López M, Méndez M, Begega A, Arias JL. Spatial short-term memory in rats: effects of learning trials on metabolic activity of limbic structures. Neurosci Lett. 2010;483:32-5.

38. González-Pardo H, Novelli A, Menéndez-Patterson A, Arias JL. The developmental of oxidative metabolism in diencephalic structures of the rat: a quantitative study. Brain Res Bull. 1996;41:31-8.

39. Paxinos G, Watson C. The rat brain in stereotaxic coordinates. 5th editio. Elsevier Academic Press; 2004.

40. Segata N, Waldron L, Ballarini A, Narasimhan V, Jousson O, Huttenhower C. Metagenomic microbial community profiling using unique clade-specific marker genes. Nat Methods. 2012;9:811-4.

41. de Souza RM, de Souza L, Machado AE, de Bem Alves AC, Rodrigues FS, Aguiar AS, et al. Behavioural, metabolic and neurochemical effects of environmental enrichment in high-fat cholesterol-enriched diet-fed mice. Behav Brain Res. 2019;359:648-56.

42. Apergis-Schoute J, Pinto A, Paré D. Ultrastructural organization of medial prefrontal inputs to the rhinal cortices. Eur J Neurosci. 2006;24:135-44.

43. Simon AJ, Skinner SN, Ziegler DA. Training working memory: anatomy matters. J Neurosci. 2016;36:7805-6.

44. Bolkan SS, Stujenske JM, Parnaudeau S, Spellman TJ, Rauffenbart C, Abbas Al, et al. Thalamic projections sustain prefrontal activity during working memory maintenance. Nat Neurosci. 
2017;20:987-96.

45. Pan X, Jiang T, Zhang L, Zheng H, Luo J, Hu X. Physical exercise promotes novel object recognition memory in spontaneously hypertensive rats after ischemic stroke by promoting neural plasticity in the entorhinal cortex. Front Behav Neurosci. 2017;11:185.

46. Tong L, Shen $\mathrm{H}$, Perreau VM, Balazs R, Cotman CW. Effects of exercise on gene-expression profile in the rat hippocampus. Neurobiol Dis. 2001;8:1046-56.

47. Mármol F, Rodríguez CA, Sánchez J, Chamizo VD. Anti-oxidative effects produced by environmental enrichment in the hippocampus and cerebral cortex of male and female rats. Brain Res. 2015;1613:120-9.

48. Putignani L, Del Chierico F, Petrucca A, Vernocchi P, Dallapiccola B. The human gut microbiota: a dynamic interplay with the host from birth to senescence settled during childhood. Pediatr Res. 2014;76:2-10.

49. Sullivan OO, Cronin O, Clarke SF, Murphy EF, Molloy MG, Shanahan F, et al. Exercise and the microbiota. Gut Microbes. 2015;6:131-6.

50. Allen JM, Mailing LJ, Niemiro GM, Moore R, Cook MD, White BA, et al. Exercise alters gut microbiota composition and function in lean and obese humans. Med Sci Sports Exerc. 2018;50:747-57.

51. Saint-Georges-Chaumet Y, Edeas M. Microbiota-mitochondria inter-talk: consequence for microbiota-host interaction. Pathog Dis. 2018;74:ftv096.

52. Kelly JR, Allen AP, Temko A, Hutch W, Kennedy PJ, Farid N, et al. Lost in translation? The potential psychobiotic Lactobacillus rhamnosus (JB-1) fails to modulate stress or cognitive performance in healthy male subjects. Brain Behav Immun. 2017;61:50-9.

53. Everard A, Belzer C, Geurts L, Ouwerkerk JP, Druart C, Bindels LB, et al. Cross-talk between Akkermansia muciniphila and intestinal epithelium controls diet-induced obesity. Proc Natl Acad Sci. 2013;110:9066-71.

54. Everard A, Plovier H, Rastelli M, Van Hul M, de Wouters d'Oplinter A, Geurts L, et al. Intestinal epithelial $\mathrm{N}$-acylphosphatidylethanolamine phospholipase $\mathrm{D}$ links dietary fat to metabolic adaptations in obesity and steatosis. Nat Commun. 2019;10:457.

55. Plovier H, Everard A, Druart C, Depommier C, Van Hul M, Geurts L, et al. A purified membrane protein from Akkermansia muciniphila or the pasteurized bacterium improves metabolism in obese and diabetic mice. Nat Med. 2017;23:107-13.

56. De Chiara F, Heebøll S, Marrone G, Montoliu C, Hamilton-Dutoit S, Ferrandez A, et al. Urea cycle dysregulation in non-alcoholic fatty liver disease. J Hepatol. 2018;69:905-15.

57. Mehrpouya-Bahrami P, Chitrala KN, Ganewatta MS, Tang C, Murphy EA, Enos RT, et al. Blockade of CB1 cannabinoid receptor alters gut microbiota and attenuates inflammation and diet-induced obesity. Sci Rep. 2017;7:15645.

58. Sharkey KA, Wiley JW. The role of the endocannabinoid system in the brain-gut axis. Gastroenterology. 2016;151:252-66. 
59. Herkenham M, Lynn AB, Litrle MD, Johnsont MR, Melvin LS, Costa BRDE, et al. Cannabinoid receptor localization in brain. Proc Natl Acad Sci U S A. 1990;87:1932-6.

60. Moreira FA, Jupp B, Belin D, Dalley JW. Endocannabinoids and striatal function: Implications for addiction-related behaviours. Behav Pharmacol. 2015;26:59-72.

61. Bénard G, Massa F, Puente N, Lourenço J, Bellocchio L, Soria-Gómez E, et al. Mitochondrial CB 1 receptors regulate neuronal energy metabolism. Nat Neurosci. 2012;15:567.

62. McEwen BS, Nasca C, Gray JD. Stress effects on neuronal structure: hippocampus, amygdala, and prefrontal cortex. Neuropsychopharmacology. 2016;41:3-23.

63. Herman JP, Ostrander MM, Mueller NK, Figueiredo H. Limbic system mechanisms of stress regulation: Hypothalamo-pituitary- adrenocortical axis. Prog Neuro-Psychopharmacology Biol Psychiatry. 2005;29:1201-13.

64. Jones $\mathrm{CP}$, Boyd KL, Wallace JM. Evaluation of mice undergoing serial oral gavage while awake or anesthetized. J Am Assoc Lab Anim Sci. 2016;55:805-10.

65. Ríos Covián D, González S, Nogacka AM, Arboleya S, Salazar N, Gueimonde M, et al. An overview on fecal branched short chain fatty acids along human life and as related with body mass index: dietary and anthropometric factors associated. Front Microbiol. :Accepted for publication on 22/4/2020.

66. Borghi E, Borgo F, Severgnini M, Savini MN, Casiraghi MC, Vignoli A. Rett syndrome: a focus on gut microbiota. Int J Mol Sci. 2017;18:344.

67. Mack I, Cuntz U, Grmer C, Niedermaier S, Pohl C, Schwiertz A, et al. Weight gain in anorexia nervosa does not ameliorate the faecal microbiota, branched chain fatty acid profiles, and gastrointestinal complaints. Sci Rep. 2016;6:26752.

68. Frost G, Sleeth ML, Sahuri-Arisoylu M, Lizarbe B, Cerdan S, Brody L, et al. The short-chain fatty acid acetate reduces appetite via a central homeostatic mechanism. Nat Commun. 2014;5:3611.

69. De Vadder F, Kovatcheva-Datchary P, Goncalves D, Vinera J, Zitoun C, Duchampt A, et al. Microbiotagenerated metabolites promote metabolic benefits via gut-brain neural circuits. Cell. 2014;156:8496.

70. Li Z, Yi CX, Katiraei S, Kooijman S, Zhou E, Chung CK, et al. Butyrate reduces appetite and activates brown adipose tissue via the gut-brain neural circuit. Gut. 2018;67:1269-79.

\section{Figures}


a

\begin{tabular}{|c|c|c|c|c|c|c|c|c|c|c|c|c|c|}
\hline W1 & W2 & W3 & W4 & W5 & W6 & W7 & W8 & W9 & W10 & W11 & W12 & W13 & W14 \\
\hline & $\begin{array}{c}D i \\
(N C / 1\end{array}$ & & & & & & & $\begin{array}{r}\text { Tre } \\
(E E / P\end{array}$ & $\begin{array}{l}\text { pent } \\
\text { biotics) }\end{array}$ & & & & uatio \\
\hline
\end{tabular}

b

C

Locomotor activity
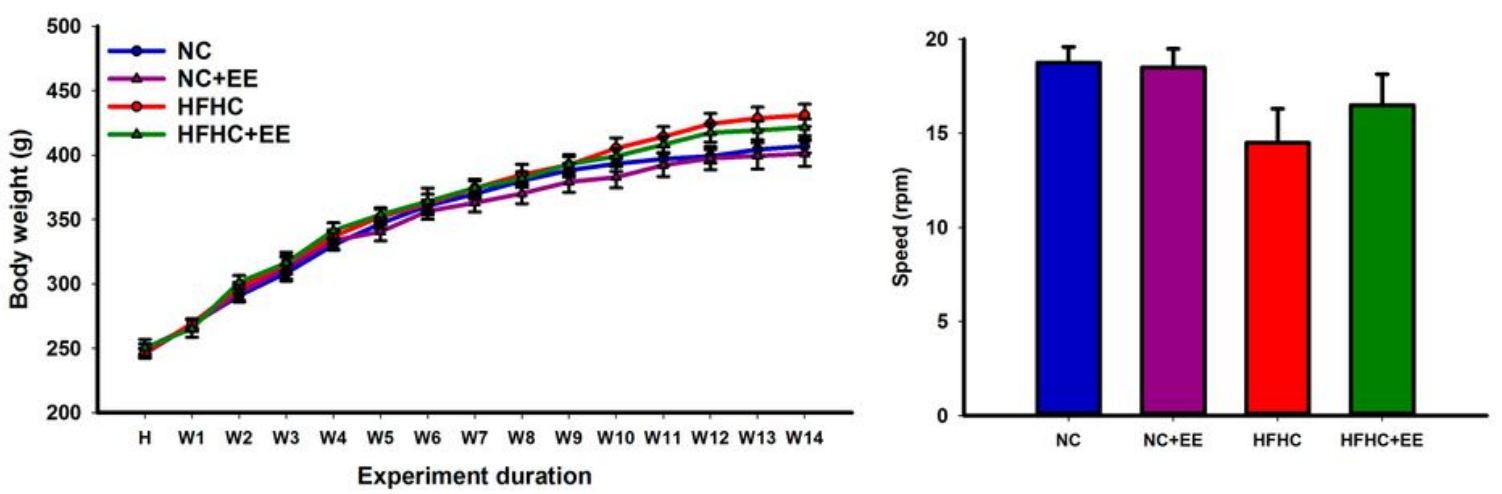

d Novel object recognition

e

Spatial working memory
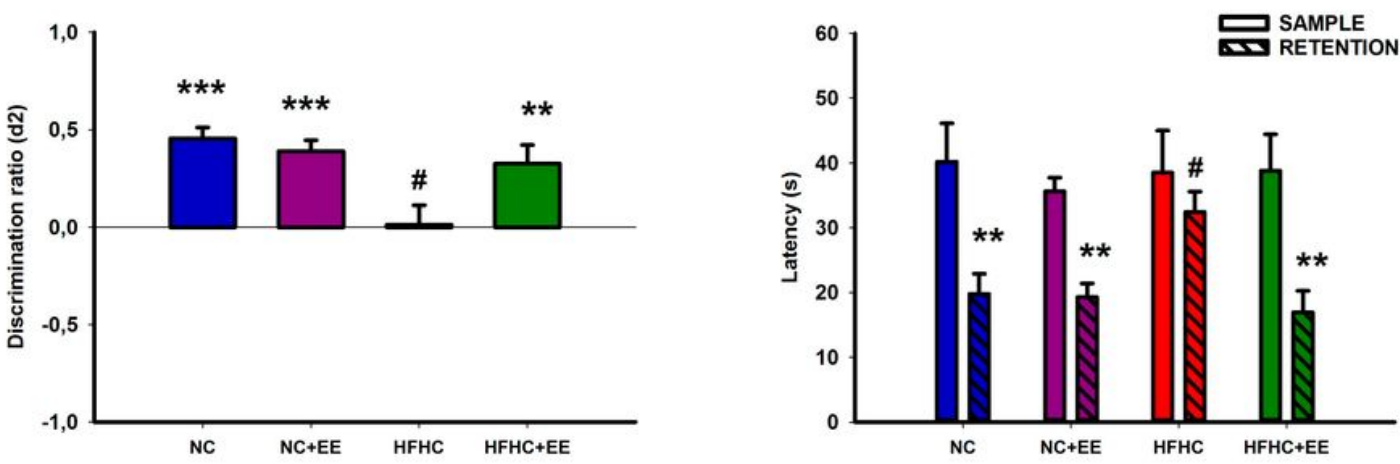

\section{Figure 1}

Timeline, body weight and behavioural assessment of the experimental groups subjected to diet and EE. a Timeline of the experimental design. 


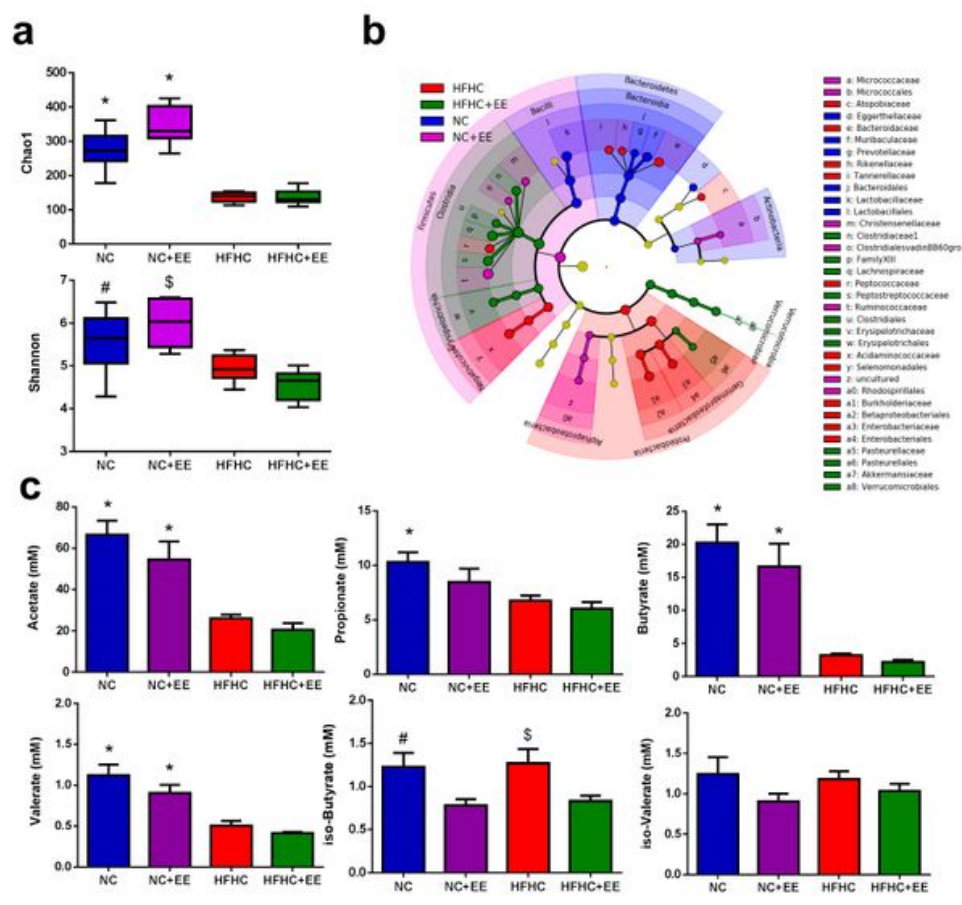

Figure 2

Gut microbiota exploration of different groups subjected to NC and HFHC diets and EE. a Bacterial diversity. 
a

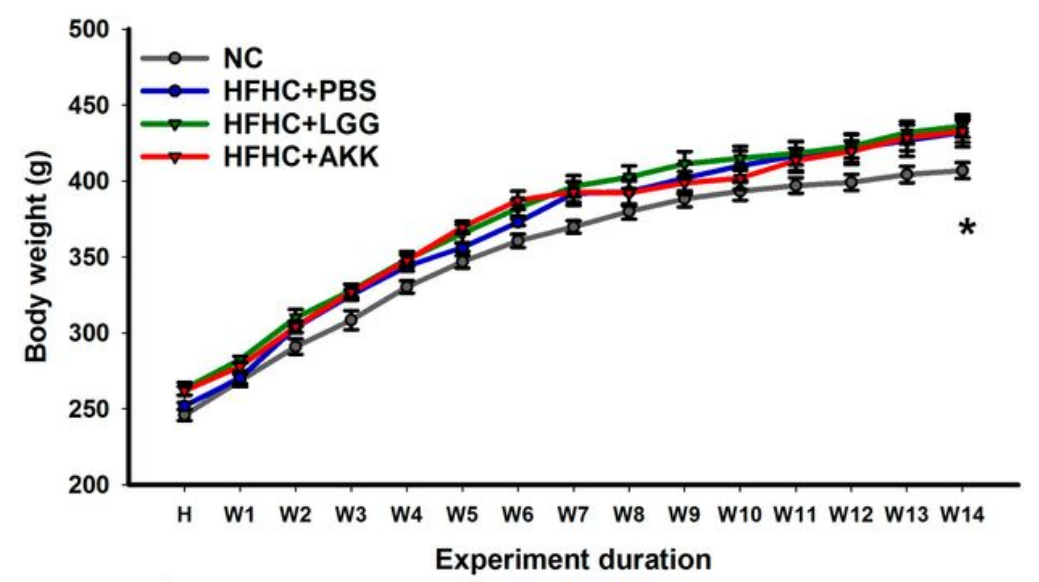

C

Novel object recognition

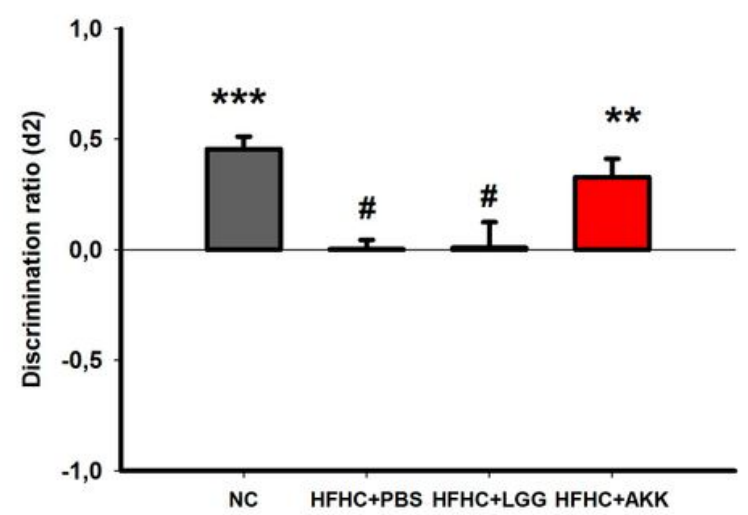

b

Locomotor activity

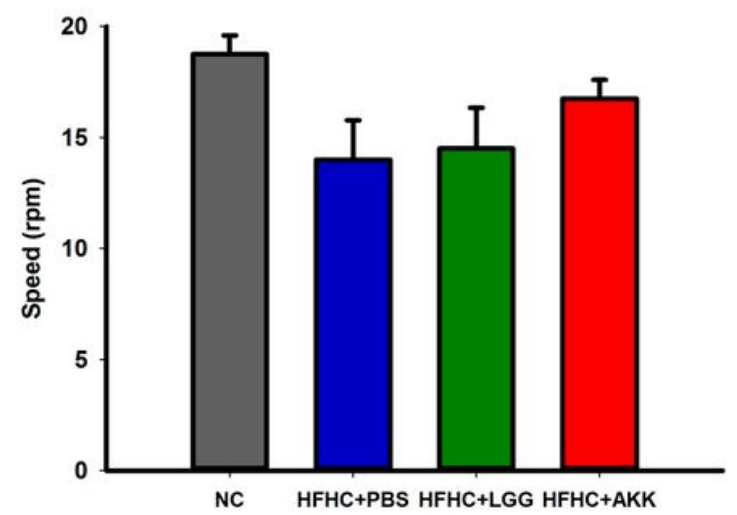

d

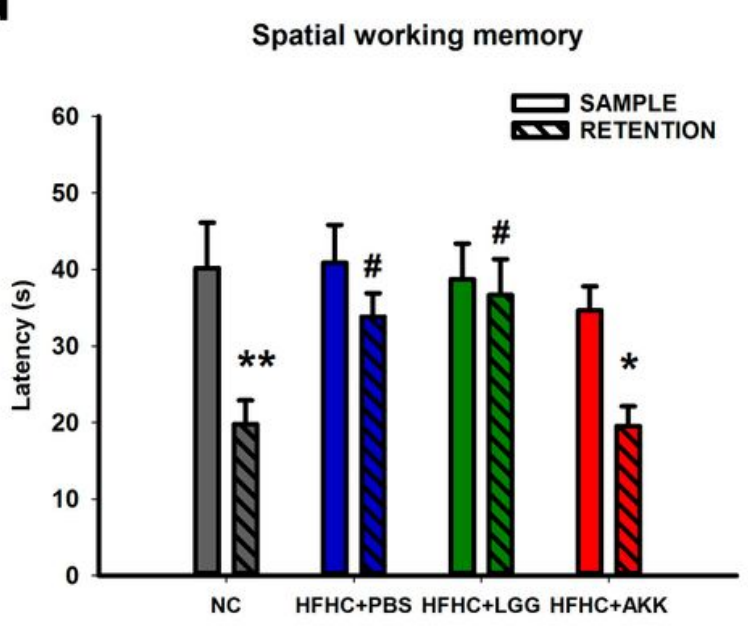

Figure 3

Body weight and behavioural assessment in experimental groups subjected to PBS, LGG and AKK. a Body weight across experimental weeks. 
a

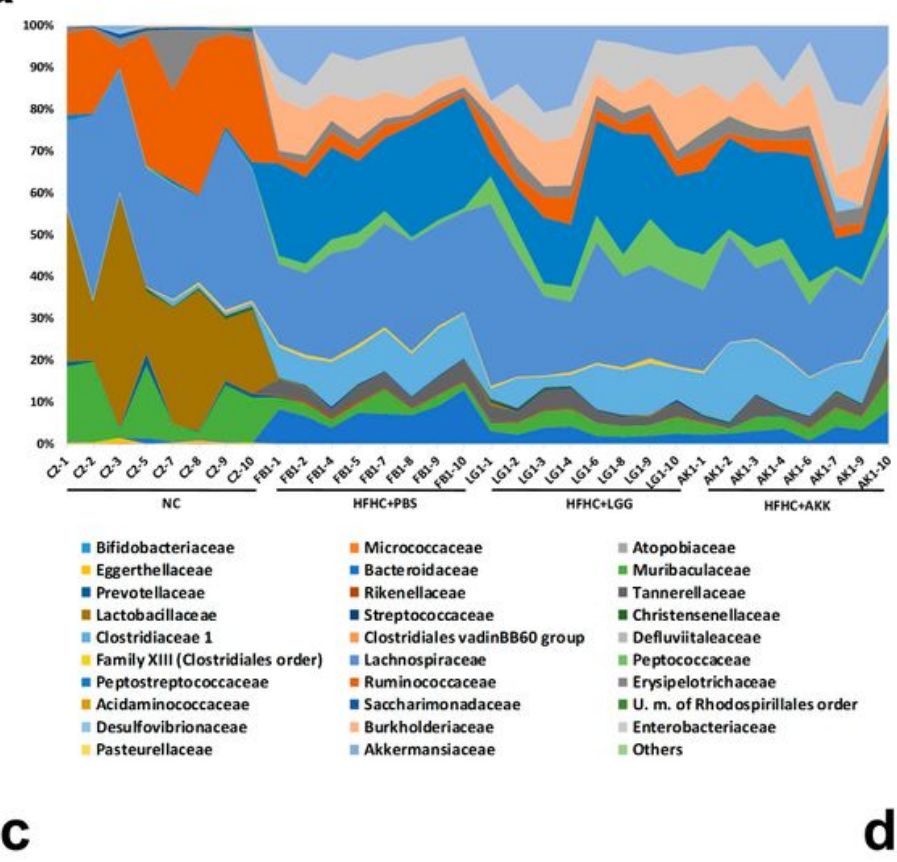

b

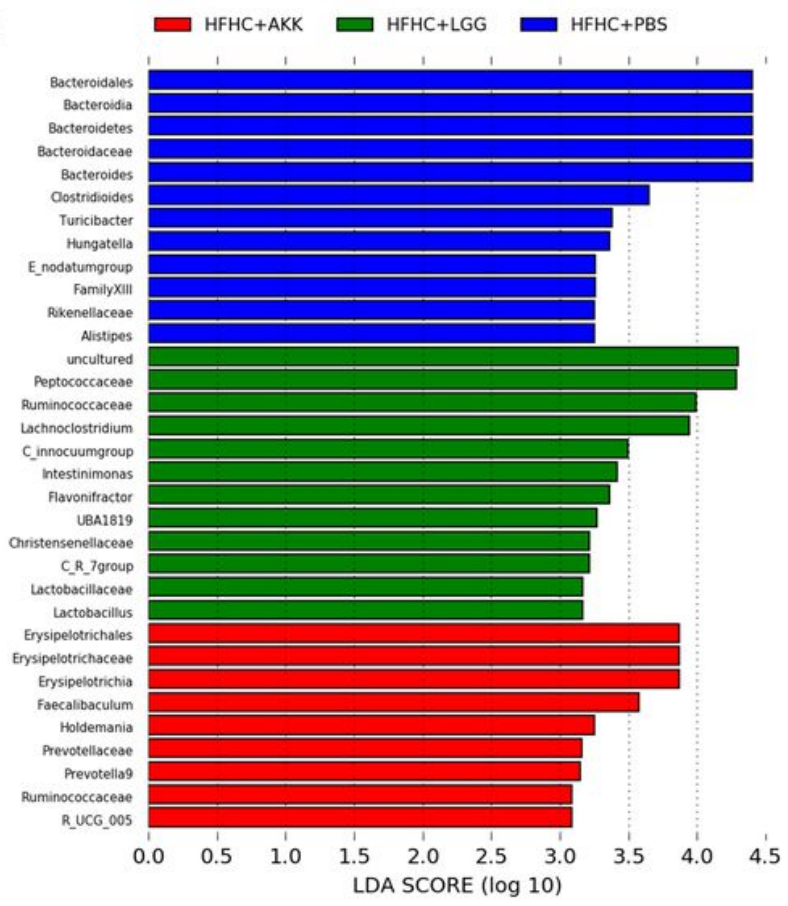

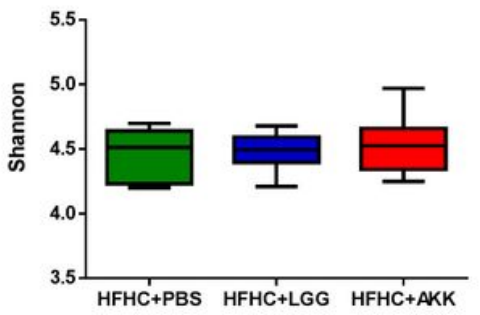

\section{Figure 4}

Gut microbiota exploration in experimental groups subjected to PBS, LGG and AKK. a Aggregate microbiota composition. 
a

Locomotor activity

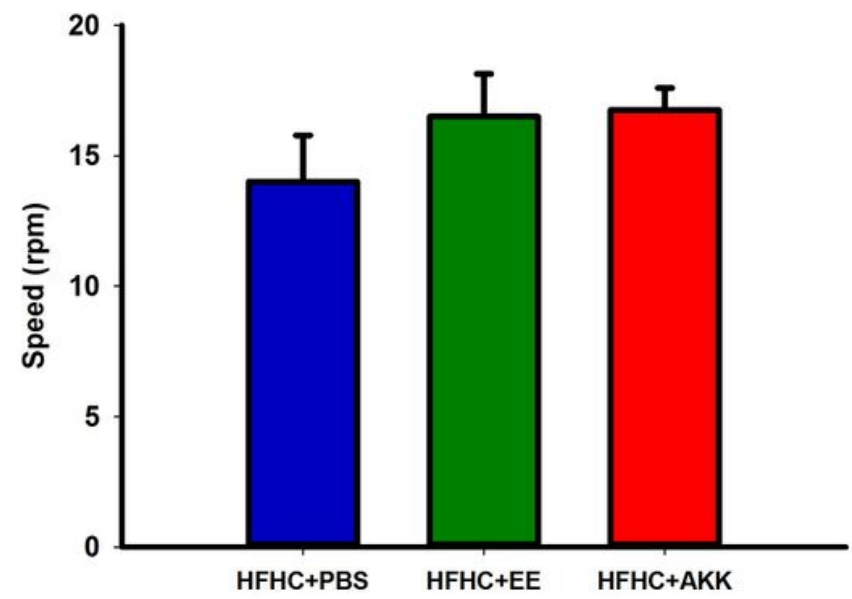

b

Novel object recognition

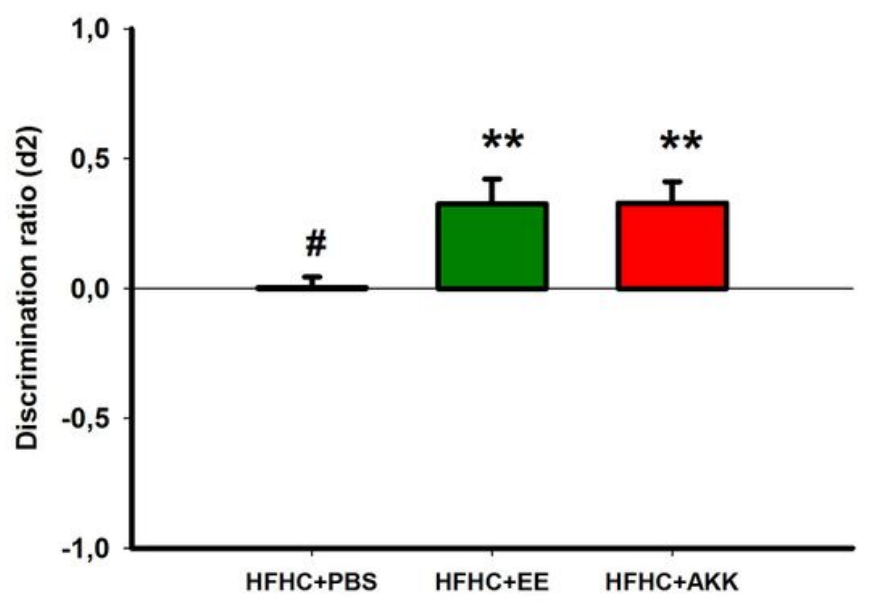

C

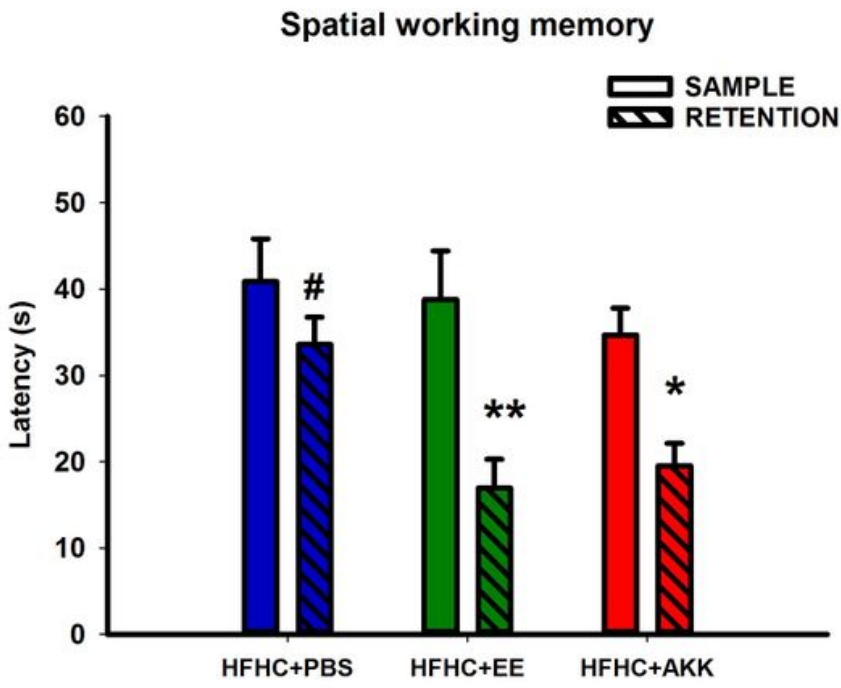

Figure 5

Behavioural assessment in different HFHC groups subjected to the administration of PBS, AKK and EE. a Locomotor function evaluation measured in Rotarod-accelerod test. 


\section{HFHC+EE}

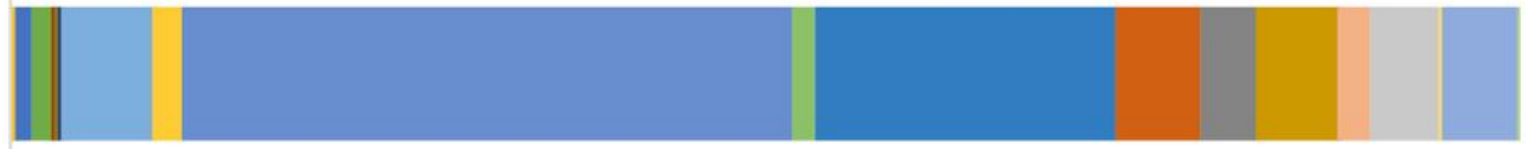

HFHC+PBS

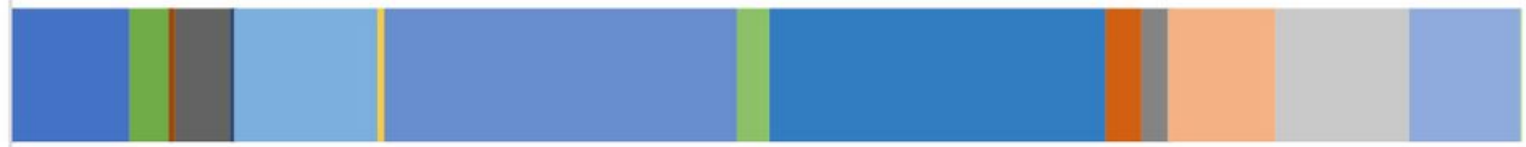

HFHC+AKK

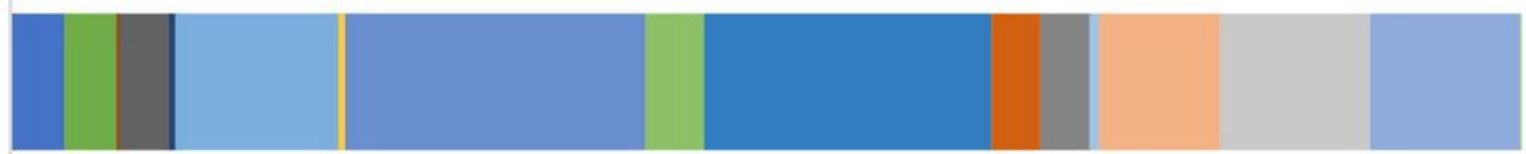

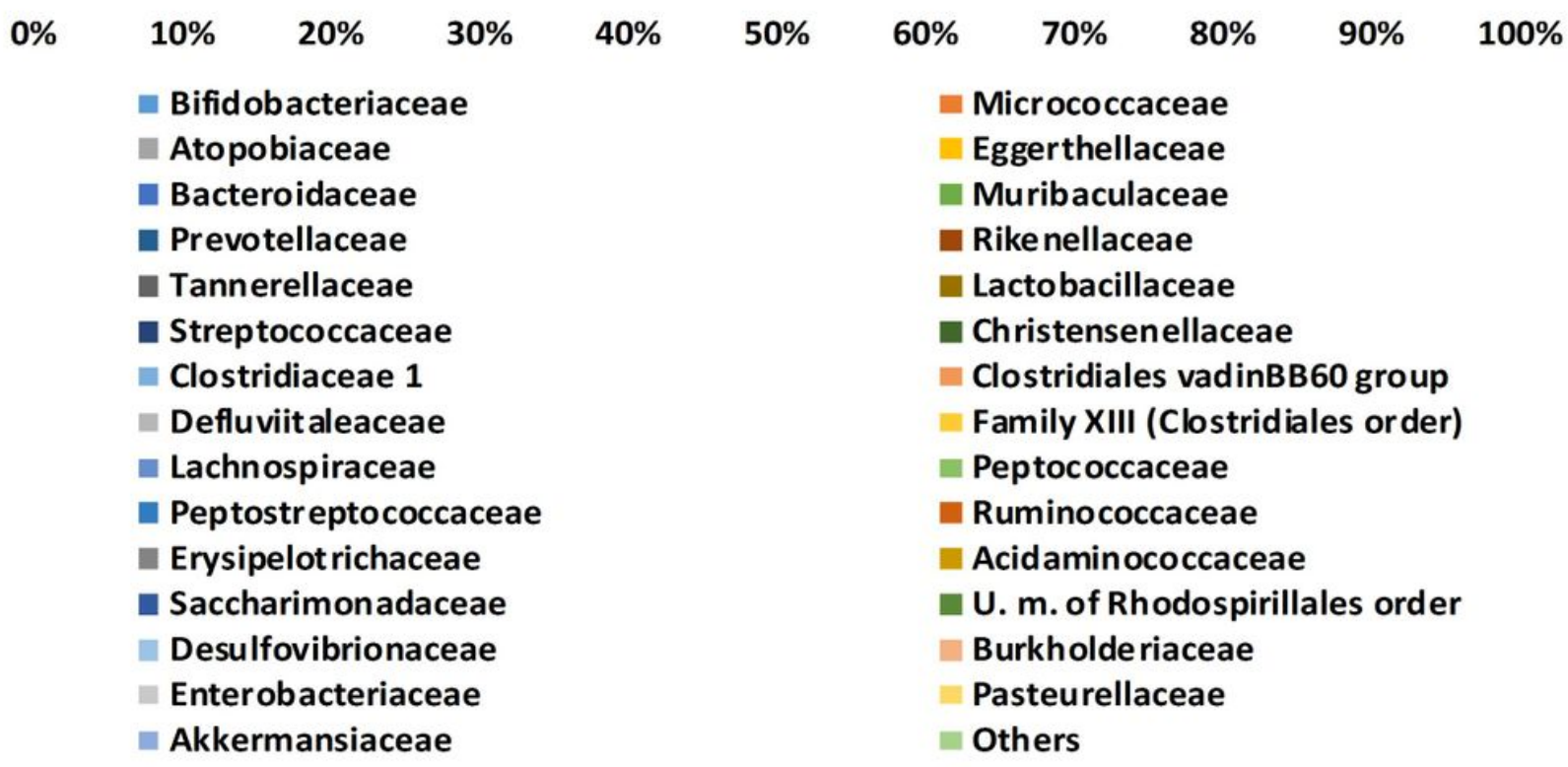

Figure 6

Gut microbiota analysis in different HFHC groups subjected to the administration of PBS, AKK and EE.

\section{Supplementary Files}

This is a list of supplementary files associated with this preprint. Click to download.

- Additionalfile1.doc 